\title{
On Complex Intuitionistic Fuzzy Soft Sets with Distance Measures and Entropies
}

\author{
Tanuj Kumar and Rakesh Kumar Bajaj \\ Department of Mathematics, Jaypee University of Information Technology, Waknaghat, Solan, Himachal Pradesh 173234, India \\ Correspondence should be addressed to Rakesh Kumar Bajaj; rakesh.bajaj@gmail.com
}

Received 21 May 2014; Revised 14 November 2014; Accepted 17 November 2014; Published 14 December 2014

Academic Editor: Hee S. Kim

Copyright (C) 2014 T. Kumar and R. K. Bajaj. This is an open access article distributed under the Creative Commons Attribution License, which permits unrestricted use, distribution, and reproduction in any medium, provided the original work is properly cited.

\begin{abstract}
We introduce the concept of complex intuitionistic fuzzy soft sets which is parametric in nature. However, the theory of complex fuzzy sets and complex intuitionistic fuzzy sets are independent of the parametrization tools. Some real life problems, for example, multicriteria decision making problems, involve the parametrization tools. In order to get their new entropies, some important properties and operations on the complex intuitionistic fuzzy soft sets have also been discussed. On the basis of some well-known distance measures, some new distance measures for the complex intuitionistic fuzzy soft sets have also been obtained. Further, we have established correspondence between the proposed entropies and the distance measures of complex intuitionistic fuzzy soft sets.
\end{abstract}

\section{Introduction}

Ramot et al. $[1,2]$ introduced a new innovative concept of complex fuzzy set (CFS), where the membership function $\mu$ instead of being a real valued function with the range of $[0,1]$ is replaced by a complex-valued function of the form $r_{A}(x) \cdot e^{i \Omega_{A}(x)}(i=\sqrt{-1})$, where $r_{A}(x)$ is a real valued function such that $r_{A}(x) \in[0,1]$ and $\Omega_{A}(x)$ is a periodic function. The key feature of complex fuzzy sets is the presence of phase and its membership. This gives those complex fuzzy sets wavelike properties which could result in constructive and destructive interference depending on the phase value. Several examples are given in [2], which demonstrate the utility of these complex fuzzy sets. They also defined several important operations such as complement, union, and intersection and discussed fuzzy relations for such complex fuzzy sets. On the other hand, Ma et al. [3] used the complex fuzzy set to represent the information with uncertainty and periodicity, where they introduced a product-sum aggregation operator based prediction (PSAOP) method to find the solution of the multiple periodic factor prediction (MPFP) problems. Further, Chen et al. [4] proposed a neurofuzzy system architecture to implement the complex fuzzy rule as a practical application of the concept of complex fuzzy logic.
Intuitionistic fuzzy set (IFS), introduced by Atanassov [5], is a controlling tool to deal with vagueness and uncertainty. A prominent characteristic of IFS is that it assigns to each element a membership degree and a nonmembership degree with certain amount of hesitation degree. Atanassov [6, 7] and many other researchers $[8,9]$ studied different properties of IFSs in decision making problems, particularly in the case of medical diagnosis, sales analysis, new product marketing, financial services, and so forth. Alkouri and Salleh [10] introduced the concept of complex intuitionistic fuzzy set (CIFS) to represent the information which is happening repeatedly over a period of time. Further, as an application, Alkouri and Salleh [11] presented an example of suppler selection model which is based on the distance measure of complex intuitionistic fuzzy sets.

Molodtsov [12] pointed out that the important existing theories, namely, probability theory, fuzzy set theory, intuitionistic fuzzy set theory, rough set theory, and so forth, which can be considered as mathematical tools for dealing with uncertainties, have their own difficulties. The inadequacy of the parametrization tools of these theories makes them very limited and difficult. In order to overcome the above-stated difficulties, Molodtsov [12] introduced the concept of soft sets for dealing with uncertainties in 
parameterized form. Later on Maji et al. [13-17] extended soft sets to fuzzy soft sets and intuitionistic fuzzy soft sets. In 2005, Pei and Miao [18] and Chen et al. [19] have studied and extended the work of Maji et al. [13,14]. Also, Majumdar and Samanta [20] have further generalized the concept of fuzzy soft sets.

In the present paper, we studied some fundamentals of soft set theory, fuzzy soft set theory, intuitionistic fuzzy soft sets, complex fuzzy sets, complex intuitionistic fuzzy sets, and some of their operations in Section 2. In Section 3, we introduced the concept of complex intuitionistic fuzzy soft sets (CIFSSs) along with their basic operations. New distance measures for CIFSSs have been obtained on the basis of some well-known distance measures and a general way to find the entropies of complex intuitionistic fuzzy soft sets has also been proposed in Section 4. An application in the area of multicriteria decision making problem on the basis of the proposed CIFSSs has also been suggested in Section 5. Finally, the paper has been concluded in Section 6 .

\section{Preliminaries}

In this section, we present some basic definitions of the soft set, fuzzy soft set, intuitionistic fuzzy soft set, complex fuzzy soft set, and some operations on them which are well known in literature $[1,2,12,15,16,21]$.

Definition 1 (see [12]). Let $X$ be the universal set and let $E$ be the set of parameters under consideration. Let $\mathscr{P}(X)$ denote the power set of $X$. A soft set may be represented by the set of ordered pairs as

$$
\langle F, E\rangle=\{\langle\varepsilon, F(\varepsilon)\rangle \mid \varepsilon \in E, F(\varepsilon) \in P(X)\},
$$

where $F$ is a mapping given by

$$
F: E \longrightarrow P(X) \text {. }
$$

In other words, the soft set is a parameterized family of subsets of the universe $X$. For each $\varepsilon \in E, F(\varepsilon)$ may be considered as a set of $\varepsilon$-elements or as a set of $\varepsilon$-approximate elements of the soft set $\langle F, E\rangle$.

Definition 2 (see [16]). Let $X$ be the universal set and let $E$ be the set of parameters under consideration. Let $\mathscr{I} \mathscr{F} \mathcal{S}(X)$ denote the set of all intuitionistic fuzzy subset of $X$. An intuitionistic fuzzy soft set $\widetilde{F}$ may be represented by the set of ordered pairs as

$$
\langle\widetilde{F}, E\rangle=\{\langle\varepsilon, \widetilde{F}(\varepsilon)\rangle \mid \varepsilon \in E, \widetilde{F}(\varepsilon) \in \mathscr{F} \mathscr{F} \mathcal{S}(X)\},
$$

where $\widetilde{F}$ is a mapping given by $\widetilde{F}: E \rightarrow \mathscr{F} \mathscr{F} \mathcal{S}(X)$ such that $\widetilde{F}(\varepsilon)=\phi$; that is, $\mu_{\widetilde{F}(\varepsilon)}(x)=0$ and $\nu_{\widetilde{F}(\varepsilon)}(x)=1$, for all $x \in X$, if $\varepsilon \notin E$.

Definition 3 (see $[16,21]$ ). Suppose that $\langle\widetilde{F}, E\rangle$ and $\langle\widetilde{G}, E\rangle$ are two intuitionistic fuzzy soft sets over a universal set $X$. Then in view of the above definition, the following operations have been defined as follows.
$(P 1)\langle\widetilde{F}, E\rangle \cup\langle\widetilde{G}, E\rangle=\langle\widetilde{H}, E\rangle$, where $\forall \varepsilon \in E, x \in X$. Consider

$\widetilde{H}(\varepsilon)$

$=\left\{\left\langle x, \mu_{\widetilde{F}(\varepsilon)}(x) \diamond \mu_{\widetilde{G}(\varepsilon)}(x), \nu_{\widetilde{F}(\varepsilon)}(x) * v_{\widetilde{G}(\varepsilon)}(x)\right\rangle \mid x \in X\right\}$.

$(P 2)\langle\widetilde{F}, E\rangle \cap\langle\widetilde{G}, E\rangle=\langle\widetilde{H}, E\rangle$, where $\forall \varepsilon \in E, x \in X$. Consider

$\widetilde{H}(\varepsilon)$

$=\left\{\left\langle x, \mu_{\widetilde{F}(\varepsilon)}(x) * \mu_{\widetilde{G}(\varepsilon)}(x), \nu_{\widetilde{F}(\varepsilon)}(x) \diamond \nu_{\widetilde{G}(\varepsilon)}(x)\right\rangle \mid x \in X\right\}$.

$(P 3)(\widetilde{F}, E)^{c}=\left(\widetilde{F}^{c}, \neg E\right)$, where mapping $\widetilde{F}^{c}: \neg E \rightarrow$ $\mathscr{I} \mathscr{F} \mathcal{S}(X)$ is given by

$$
\begin{aligned}
\widetilde{F}^{c}(\neg \mathcal{E}) & =\left\{\left\langle x, \nu_{\widetilde{F}(\neg \neg \varepsilon)}(x), \mu_{\widetilde{F}(\neg \neg \varepsilon)}(x)\right\rangle \mid x \in X\right\} \\
& =\left\{\left\langle x, \nu_{\widetilde{F}(\varepsilon)}(x), \mu_{\widetilde{F}(\varepsilon)}(x)\right\rangle \mid x \in X\right\}, \quad \forall \neg \varepsilon \in \neg E .
\end{aligned}
$$

$(P 4)\langle\widetilde{F}, E\rangle \subseteq\langle\widetilde{G}, E\rangle$, if and only if $\mu_{\widetilde{F}(\varepsilon)}(x) \leq \mu_{\widetilde{G}(\varepsilon)}(x)$ and $\nu_{\widetilde{F}(\varepsilon)}(x) \geq \nu_{\widetilde{G}(\varepsilon)}(x), \forall x \in X$, and $\varepsilon \in E$.

$(P 5)\langle\widetilde{F}, E\rangle=\langle\widetilde{G}, E\rangle$, if and only if $\mu_{\widetilde{F}(\varepsilon)}(x)=\mu_{\widetilde{G}(\varepsilon)}(x)$ and $\nu_{\widetilde{F}(\varepsilon)}(x)=\nu_{\widetilde{G}(\varepsilon)}(x), \forall x \in X$, and $\varepsilon \in E$,

where $\diamond$ and $*$ are $s$-norm and $t$-norm operators, respectively.

Definition 4 (see [1]). A complex fuzzy set $A$, defined on universal set $X$, is characterized by the membership function $\mu_{A}(x)$, which assign to each element $x \in X$ a complex-valued grade of membership in $A$.

Let $\mathscr{C} \mathscr{F} \mathcal{S}(X)$ be the set of all complex fuzzy sets on $X$. The complex fuzzy set $A$ may be represented as the set of ordered pairs:

$$
A=\left\{\left\langle x, \mu_{A}(x)\right\rangle: x \in X\right\}
$$

where $\mu_{A}(x): X \rightarrow\{a|a \in \mathbb{C}| a \mid, \leq 1\}$.

By definition, the values of $\mu_{A}(x)$ may receive all the values lying within the unit circle in the complex plane and are of the form

$$
\mu_{A}(x)=r_{A}(x) \cdot e^{i \Omega_{A}(x)}, \quad(i=\sqrt{-1}),
$$

where $r_{A}$ is a real valued function such that $r_{A}(x) \in[0,1]$ and $\Omega_{A}$ is a periodic function whose periodic law and principal period are, respectively, $2 \pi$ and $0 \leq \omega_{A}(x) \leq 2 \pi$; that is,

$$
\Omega_{A}(x)=\omega_{A}(x)+2 k \pi, \quad k=0, \pm 1, \pm 2, \ldots,
$$

where $\omega_{A}(x)$ is the principal argument.

Definition 5 (see [2]). Let $A$ and $B$ be two complex fuzzy sets on $X$, and $\mu_{A}(x)=r_{A}(x) \cdot e^{i \omega_{A}(x)}$ and $\mu_{B}(x)=r_{B}(x) \cdot e^{i \omega_{B}(x)}$ are 
their membership functions, respectively. Then the following operations have been defined as follows:

(a) union: $A \bigcup B=\left\{\left\langle x, r_{A}(x) \diamond r_{B}(x) \cdot e^{\omega_{A \cup B}(x)}\right\rangle \mid x \in X\right\}$,

(b) intersection: $A \bigcap B=\left\{\left\langle x, r_{A}(x) * r_{B}(x) \cdot e^{\omega_{A \cup B}(x)}\right\rangle\right.$ | $x \in X\}$,

where the $\diamond$ and $*$ are $s$-norm and $t$-norm operators, respectively. It remains to define $\omega_{A \cup B}(x)$ and $\omega_{A \cap B}(x)$ for which the following axioms have to be followed.

Definition 6 (see [2]). Let $A$ and $B$ be two complex fuzzy sets in $X$. Then complex fuzzy union and intersection are specified by the functions $u$ and $v$ as follows:

$$
\begin{aligned}
& u:\{a|a \in \mathbb{C},| a \mid \leq 1\} \times\{b|b \in \mathbb{C},| b \mid \leq 1\} \\
& \quad \longrightarrow\{c|c \in \mathbb{C},| c \mid \leq 1\}, \\
& v:\{a|a \in \mathbb{C},| a \mid \leq 1\} \times\{b|b \in \mathbb{C},| b \mid \leq 1\} \\
& \quad \longrightarrow\{c|c \in \mathbb{C},| c \mid \leq 1\} ;
\end{aligned}
$$

$u$ must satisfy at least the following axioms requirements:

(i) (boundary conditions): $u(a, 0)=a$;

(ii) (monotonicity): $|b| \leq|d| \Rightarrow|u(a, b)| \leq|u(a, d)|$;

(iii) (commutativity): $u(a, b)=u(b, a)$;

(iv) (associativity): $u(a, u(b, d))=u(u(a, b), d)$.

In some cases, it may be desirable that $u$ also satisfies the following requirements:

(i) (continuity): $u$ is a continuous function;

(ii) (superidempotency): $|u(a, a)|>|a|$;

(iii) (strict monotonicity): $|a| \leq|c|$ and $|b| \leq|d| \Rightarrow$ $|u(a, b)| \leq|u(c, d)|$.

And $v$ must satisfy the following axiomatic requirements:

(i) (boundary conditions): if $|b|=1$, then $v(a, b)=|a|$;

(ii) (monotonicity): $|b| \leq|d| \Rightarrow|v(a, b)| \leq|v(a, d)|$;

(iii) (commutativity): $v(a, b)=v(b, a)$;

(iv) (associativity): $v(a, v(b, d))=v(v(a, b), d)$.

In some cases, it may be required that $v$ also satisfies the following axioms:

(i) (continuity): $v$ is a continuous function;

(ii) (superidempotency): $|v(a, a)|<|a|$;

(iii) (strict monotonicity): $|a| \leq|c|$ and $|b| \leq|d| \Rightarrow$ $|v(a, b)| \leq|v(c, d)|$.

The following are several possibilities for calculation of $\omega_{A \cup B}(x)$ and $\omega_{A \cap B}(x)$, which, if combined with an appropriate function for determining $r_{A \cup B}(x)$ and $r_{A \cap B}(x)$, satisfies the axiomatic requirements given in the above definitions.

(1) Sum: $\omega_{A}(x)+\omega_{B}(x)$.
(2) $\operatorname{Max}: \max \left(\omega_{A}(x), \omega_{B}(x)\right)$.

(3) Min: $\min \left(\omega_{A}(x), \omega_{B}(x)\right)$.

(4) "Winner Take All": $\begin{cases}\omega_{A}(x), & \text { if } r_{A}(x)>r_{B}(x) \\ \omega_{B}(x), & \text { if } r_{A}(x) \leq r_{B}(x) .\end{cases}$

Let $C$ be a complex fuzzy set on $X$ with the membership function $\mu_{C}(x)=r_{C}(x) \cdot e^{i \omega_{C}(x)}$. The complex fuzzy complement of $C$, denoted by $\bar{C}$, is specified by a function

$$
\mu_{\bar{C}}(x)=\left(1-r_{C}(x)\right) \cdot e^{i \omega_{\bar{C}}(x)} .
$$

Ramot et al. [1] obtained several possible methods for calculating the membership phase of complex fuzzy complement, $\omega_{\bar{C}}(x)$. For example, $\omega_{\bar{C}}(x)$ is defined as $\omega_{\bar{C}}(x)=\omega_{C}(x)$; also $\omega_{\overline{\mathrm{C}}}(x)$ may be defined by the relation $\omega_{\overline{\mathrm{C}}}(x)=2 \pi-$ $\omega_{C}(x)$, where Zhang et al. [22] used this relation to define the complement for the phase component; also the rotation of $\omega_{C}(x)$ by $\pi$ radians may be a good method to calculate the complement for a phase term as $\omega_{\bar{C}}(x)=\pi+\omega_{C}(x)$.

Alkouri and Salleh $[10,11]$ gave the generalization of complex fuzzy set to the complex intuitionistic fuzzy set by adding the nonmembership term to the definition of CFS. The ranges of values are extended to the unit circle in complex plane for both membership and nonmembership functions instead of $[0,1]$ as in the conventional intuitionistic fuzzy sets.

Definition 7 (see $[10,11]$ ). A complex intuitionistic fuzzy set $\widetilde{A}$, defined on a universal set $X$, is characterized by the membership and nonmembership functions $\mu_{\widetilde{A}}(x)$ and $\nu_{\widetilde{A}}(x)$, respectively, which assign to each element $x \in X$ a complex-valued grade of membership and nonmembership in $\widetilde{A}$.

Let $\mathscr{C} \mathscr{F} \mathscr{F} \mathcal{S}(X)$ be the set of all complex intuitionistic fuzzy sets on $X$. The complex intuitionistic fuzzy set $\widetilde{A}$ may be represented as the set of triplets

$$
\widetilde{A}=\left\{\left\langle x, \mu_{\widetilde{A}}(x), \nu_{\widetilde{A}}(x)\right\rangle: x \in X\right\},
$$

where $\mu_{\widetilde{A}}(x): X \rightarrow\{a|a \in \mathbb{C}| a \mid, \leq 1\}$ and $\nu_{\widetilde{A}}(x): X \rightarrow$ $\left\{a^{\prime}\left|a^{\prime} \in \mathbb{C},\right| a^{\prime} \mid \leq 1\right\}$.

By definition, the values of $\mu_{\widetilde{A}}(x), \nu_{\widetilde{A}}(x)$ and their sum all are lying within the unit circle in the complex plane and are of the form $\mu_{\widetilde{A}}(x)=r_{\widetilde{A}}(x) \cdot e^{i \omega_{\widetilde{A}}^{r}(x)}$ and $\nu_{\widetilde{A}}(x)=k_{\widetilde{A}}(x)$. $e^{i \omega_{\widetilde{A}}^{k}(x)}(i=\sqrt{-1}) ; r_{\widetilde{A}}(x), k_{\widetilde{A}}(x)$ are real valued functions such that $r_{\widetilde{A}}(x), k_{\widetilde{A}}(x) \in[0,1]$ and satisfies the condition $0 \leq r_{\widetilde{A}}(x)+k_{\widetilde{A}}(x) \leq 1$. The phase terms $\omega_{\widetilde{A}}^{r}(x)$ and $\omega_{\widetilde{A}}^{s}(x)$ belong to $(0,2 \pi]$.

\section{Complex Intuitionistic Fuzzy Soft Sets}

The concept of soft set theory [12] has been used as a generic mathematical tool for dealing with uncertainty. However, in literature, it has been pointed out that the classical soft sets are not appropriate to deal with imprecise and fuzzy parameters. In order to handle such information, the concept of fuzzy soft sets, intuitionistic fuzzy soft sets, and interval valued fuzzy soft sets have been laid down. In this section, we extend and 
introduce the concept of complex intuitionistic fuzzy soft set (CIFSS) with some important operations and properties.

Definition 8. Let $X$ be universal set and let $E$ be the set of parameters under consideration. Let $\mathscr{C} \mathscr{F} \mathscr{F} \mathcal{S}(X)$ denote the set of all complex intuitionistic fuzzy subset of $X$. A complex intuitionistic fuzzy soft set (CIFSS) may be represented by the set of ordered pairs as

$$
\langle\widetilde{F}, E\rangle=\{\langle\varepsilon, \widetilde{F}(\varepsilon)\rangle \mid \varepsilon \in E, \widetilde{F}(\varepsilon) \in \mathscr{C} \mathscr{I} \mathscr{F} \mathcal{S}(X)\},
$$

where $\widetilde{F}: E \rightarrow \mathscr{C} \mathscr{F} \mathscr{F} \mathcal{S}(X)$ such that $\widetilde{F}(\varepsilon)=\phi$ (i.e., $\mu_{\widetilde{F}(\varepsilon)}(x)=0$ and $\nu_{\widetilde{F}(\varepsilon)}(x)=1$ for all $\left.x \in X\right)$, if $\varepsilon \notin E$.

Definition 9. Suppose that $\langle\widetilde{F}, E\rangle$ and $\langle\widetilde{G}, E\rangle$ are two CIFSSs over the universal set $X$. Then in view of the above definition, one defines the following operations as follows.

$(P 1)\langle\widetilde{F}, E\rangle \cup\langle\widetilde{G}, E\rangle=\langle\widetilde{H}, E\rangle$, where $\forall x \in X, \varepsilon \in E$,

$\widetilde{H}(\varepsilon)$

$$
=\left\{\left\langle x, \mu_{\widetilde{F}(\varepsilon)}(x) \diamond \mu_{\widetilde{G}(\varepsilon)}(x), \nu_{\widetilde{F}(\varepsilon)}(x) * \nu_{\widetilde{G}(\varepsilon)}(x)\right\rangle \mid x \in X\right\} .
$$

$(P 2)\langle\widetilde{F}, E\rangle \cap\langle\widetilde{G}, E\rangle=\langle\widetilde{H}, E\rangle$, where $\forall x \in X, \varepsilon \in E$,

$$
\begin{aligned}
\widetilde{H}(\varepsilon) & \\
= & \left\{\left\langlex, \mu_{\widetilde{F}(\varepsilon)}(x) * \mu_{\widetilde{G}(\varepsilon)}(x),\right.\right. \\
& \left.\left.\nu_{\widetilde{F}(\varepsilon)}(x) \diamond \nu_{\widetilde{G}(\varepsilon)}(x)\right\rangle \mid x \in X\right\} .
\end{aligned}
$$

$(P 3)(\widetilde{F}, E)^{c}=\left(\widetilde{F}^{c}, \neg E\right)$, where $\widetilde{F}^{c}: \neg E \rightarrow \mathscr{F} \mathscr{F} \mathcal{S}(X)$ is mapping given by

$$
\begin{aligned}
\widetilde{F}^{c}(\neg \mathcal{\varepsilon}) & =\left\{\left\langle x, \nu_{\widetilde{F}(\neg \neg \varepsilon)}(x), \mu_{\widetilde{F}(\neg \neg \varepsilon)}(x)\right\rangle \mid x \in X\right\} \\
& =\left\{\left\langle x, \nu_{\widetilde{F}(\varepsilon)}(x), \mu_{\widetilde{F}(\varepsilon)}(x)\right\rangle \mid x \in X\right\}, \quad \forall \neg \varepsilon \in \neg E .
\end{aligned}
$$

$(P 4)\langle\widetilde{F}, E\rangle \subseteq\langle\widetilde{G}, E\rangle$, if and only if $\mu_{\widetilde{F}(\varepsilon)}(x) \leq \mu_{\widetilde{G}(\varepsilon)}(x)$ and $\nu_{\widetilde{F}(\varepsilon)}(x) \geq \nu_{\widetilde{G}(\varepsilon)}(x), \forall x \in X$ and $\varepsilon \in E$.

(P5) $\langle\widetilde{F}, E\rangle=\langle\widetilde{G}, E\rangle$, if and only if $\mu_{\widetilde{F}(\varepsilon)}(x)=\mu_{\widetilde{G}(\varepsilon)}(x)$ and $\nu_{\widetilde{F}(\varepsilon)}(x)=\nu_{\widetilde{G}(\varepsilon)}(x), \forall x \in X$ and $\varepsilon \in E$.

In order to propose the intuitionistic entropy of complex intuitionistic fuzzy soft sets, we need to introduce some important properties of complex intuitionistic fuzzy soft sets.

Definition 10. Let $E$ be the set of parameters and suppose that $\langle\widetilde{F}, E\rangle$ and $\langle\widetilde{G}, E\rangle$ are two complex intuitionistic fuzzy soft sets over the universal set $X$, and then one says that $\langle\widetilde{G}, E\rangle$ is a sharpened version of $\langle\widetilde{F}, E\rangle$; that is, $\langle\widetilde{F}, E\rangle \preceq\langle\widetilde{G}, E\rangle$ if and only if $\mu_{\widetilde{F}(\varepsilon)}(x) \leq \mu_{\widetilde{G}(\varepsilon)}(x)$ and $\nu_{\widetilde{F}(\varepsilon)}(x) \leq \nu_{\widetilde{G}(\varepsilon)}(x)$ (i.e., $r_{\widetilde{F}(\varepsilon)}(x) \leq r_{\widetilde{G}(\varepsilon)}(x)$ and $k_{\widetilde{F}(\varepsilon)}(x) \leq k_{\widetilde{G}(\varepsilon)}(x)$, for the amplitude terms and for the phase terms $\omega_{\widetilde{F}(\varepsilon)}^{r}(x) \leq \omega_{\widetilde{G}(\varepsilon)}^{r}(x)$ and $\left.\omega_{\widetilde{F}(\varepsilon)}^{k}(x) \leq \omega_{\widetilde{G}(\varepsilon)}^{k}(x)\right), \forall x \in X$ and $\forall \varepsilon \in E$.
Definition 11. To every element $f \in\{c|c \in \mathbb{C}| c \mid, \leq$ $1\}^{\{a|a \in \mathbb{C},| a \mid \leq 1\} \times\{b|b \in \mathbb{C},| b \mid \leq 1\}}$ one can associate a mapping $f_{\alpha}$ of $\mathscr{C} \mathscr{F} \mathscr{F} \mathcal{S} \mathcal{S}(X)$, in $\mathscr{C} \mathscr{F} \mathcal{S} \mathcal{S}(X)$, with $\alpha \in[0,1]$, given by $f_{\alpha}$ : $\langle\widetilde{F}, E\rangle \rightarrow\left\langle F_{\alpha}, E\right\rangle$, where $F_{\alpha}$ is defined as follows.

Let $\widetilde{F}(\varepsilon)=\left\{\left\langle x, \mu_{\widetilde{F}(\varepsilon)}, \nu_{\widetilde{F}(\varepsilon)}\right\rangle \mid x \in X\right\}$, and

$$
\begin{aligned}
& F_{\alpha}(\varepsilon)=f_{\alpha}(\widetilde{F}(\varepsilon)) \\
&=f_{\alpha}\left(\left\{\left\langle x, \mu_{\widetilde{F}(\varepsilon)}(x), \nu_{\widetilde{F}(\varepsilon)}(x)\right\rangle \mid x \in X\right\}\right) \\
&=\left\{\left\langlex, \mu_{F(\varepsilon)}(x)\right.\right. \\
&\left.\left.=r_{F(\varepsilon)}(x) \cdot e^{i 2 \pi \omega_{F(\varepsilon)}^{r}(x)}\right\rangle \mid x \in X\right\}, \\
& \forall \varepsilon \in E,
\end{aligned}
$$

where

$$
\begin{aligned}
& r_{F(\varepsilon)}(x)=\left(r_{\widetilde{F}(\varepsilon)}(x)+\alpha \cdot\left(1-r_{\widetilde{F}(\varepsilon)}(x)-k_{\widetilde{F}(\varepsilon)}(x)\right)\right) \\
& \omega_{F(\varepsilon)}^{r}(x) \\
& =\left[\left(\frac{\omega_{\widetilde{F}(\varepsilon)}^{r}(x)}{2 \pi}\right)+\alpha \cdot\left(1-\frac{\omega_{\widetilde{F}(\varepsilon)}^{r}(x)+\omega_{\widetilde{F}(\varepsilon)}^{k}(x)}{2 \pi}\right)\right] .
\end{aligned}
$$

The proposed operator $f_{\alpha}$ defined in Definition 11 is to assign a complex intuitionistic fuzzy soft set to a complex fuzzy soft set. The following theorem provides the properties of the operator $f_{\alpha}$.

Theorem 12. For all $\alpha, \beta \in[0,1], \xi, \tilde{\xi} \in \mathscr{C} \mathscr{F} \mathscr{F} \mathcal{S} \mathcal{S}(X)$, one has the following properties:

(1) if $\alpha \leq \beta$, then $f_{\alpha}(\xi) \subseteq f_{\beta}(\xi)$;

(2) if $\xi \subseteq \widetilde{\xi}$, then $f_{\alpha}(\xi) \subseteq f_{\alpha}(\widetilde{\xi})$;

(3) $f_{\alpha}\left(f_{\beta}(\xi)\right)=f_{\beta}(\xi)$;

(4) $\left(f_{\alpha}\left(\xi^{c}\right)\right)^{c}=f_{1-\alpha}(\xi)$.

Proof. Let $\xi=\langle\widetilde{F}, E\rangle=\left[a_{i j}\right]_{m \times n}$ and $\widetilde{\xi}=\langle\widetilde{G}, E\rangle=\left[b_{i j}\right]_{m \times n}$ be two complex intuitionistic fuzzy soft sets. Let $f_{\alpha}(\langle\widetilde{F}, E\rangle)=$ $\left\langle F_{\alpha}, E\right\rangle$, where $\widetilde{F}(\varepsilon)=\left\{\left\langle x, \mu_{\widetilde{F}(\varepsilon)}, \nu_{\widetilde{F}(\varepsilon)}\right\rangle \mid x \in X\right\}$, and

$$
\begin{aligned}
F_{\alpha}(\varepsilon) & \\
& =f_{\alpha}(\widetilde{F}(\varepsilon)) \\
& =f_{\alpha}\left(\left\{\left\langle x, \mu_{\widetilde{F}(\varepsilon)}(x), v_{\widetilde{F}(\varepsilon)}(x)\right\rangle \mid x \in X\right\}\right) \\
& =\left\{\left\langle x, r_{F_{\alpha}(\varepsilon)}(x) \cdot e^{i 2 \pi \omega_{F_{\alpha}(\varepsilon)}^{r}(x)}\right\rangle \mid x \in X\right\}, \quad \forall \varepsilon \in E .
\end{aligned}
$$


Here

$$
\begin{aligned}
& r_{F_{\alpha}(\varepsilon)}(x)=\left(r_{\widetilde{F}(\varepsilon)}(x)+\alpha \cdot\left(1-r_{\widetilde{F}(\varepsilon)}(x)-k_{\widetilde{F}(\varepsilon)}(x)\right)\right) ; \\
& \omega_{F_{\alpha}(\varepsilon)}^{r}(x) \\
& =\left[\left(\frac{\omega_{\widetilde{F}(\varepsilon)}^{r}(x)}{2 \pi}\right)+\alpha \cdot\left(1-\frac{\omega_{\widetilde{F}(\varepsilon)}^{r}(x)+\omega_{\widetilde{F}(\varepsilon)}^{k}(x)}{2 \pi}\right)\right] .
\end{aligned}
$$

Let $f_{\beta}(\langle\widetilde{F}, E\rangle)=\left\langle F_{\beta}, E\right\rangle$, where $\widetilde{F}(\varepsilon)=\left\{\left\langle x, \mu_{\widetilde{F}(\varepsilon)}, \nu_{\widetilde{F}(\varepsilon)}\right\rangle \mid x \in\right.$ $X\}$, and

$$
\begin{aligned}
F_{\beta} & (\varepsilon) \\
& =f_{\beta}(\widetilde{F}(\varepsilon)) \\
& =f_{\beta}\left(\left\{\left\langle x, \mu_{\widetilde{F}(\varepsilon)}(x), v_{\widetilde{F}(\varepsilon)}(x)\right\rangle \mid x \in X\right\}\right) \\
& =\left\{\left\langle x, r_{F_{\beta}(\varepsilon)}(x) \cdot e^{i 2 \pi \omega_{F_{\beta}(\varepsilon)}^{r}(x)}\right\rangle \mid x \in X\right\}, \quad \forall \varepsilon \in E .
\end{aligned}
$$

Here

$$
\begin{aligned}
& r_{F_{\beta}(\varepsilon)}(x)=\left(r_{\widetilde{F}(\varepsilon)}(x)+\beta \cdot\left(1-r_{\widetilde{F}(\varepsilon)}(x)-k_{\widetilde{F}(\varepsilon)}(x)\right)\right) ; \\
& \omega_{F_{\beta}(\varepsilon)}^{r}(x) \\
& \quad=\left[\left(\frac{\omega_{\widetilde{F}(\varepsilon)}^{r}(x)}{2 \pi}\right)+\beta \cdot\left(1-\frac{\omega_{\widetilde{F}(\varepsilon)}^{r}(x)+\omega_{\widetilde{F}(\varepsilon)}^{k}(x)}{2 \pi}\right)\right] .
\end{aligned}
$$

(1) In the following, we have to prove that $\mu_{F_{\alpha}(\varepsilon)}(x) \leq$ $\mu_{F_{\beta}(\varepsilon)}(x)$; that is, $r_{F_{\alpha}(\varepsilon)}(x) \leq r_{F_{\beta}(\varepsilon)}(x)$ and $\omega_{F_{\alpha}(\varepsilon)}^{r}(x) \leq$ $\omega_{F_{\beta}(\varepsilon)}^{r}(x), \forall x \in X$ and $\varepsilon \in E$. Since $\alpha \leq \beta$, therefore, we have $r_{F_{\alpha}(\varepsilon)}(x) \leq r_{F_{\beta}(\varepsilon)}(x)$ and $\omega_{F_{\alpha}(\varepsilon)}^{r}(x) \leq \omega_{F_{\beta}(\varepsilon)}^{r}(x)$, $\Rightarrow \mu_{F_{\alpha}(\varepsilon)}(x) \leq \mu_{F_{\beta}(\varepsilon)}(x), \forall x \in X$ and $\varepsilon \in E$.

Hence, $f_{\alpha}(\xi) \subseteq f_{\beta}(\xi)$.

(2) Let $f_{\alpha}(\widetilde{\xi})=f_{\alpha}(\langle\widetilde{G}, E\rangle)=\left\langle G_{\alpha}, E\right\rangle$, where $\widetilde{G}(\varepsilon)=$ $\left\{\left\langle x, \mu_{\widetilde{G}(\varepsilon)}, v_{\widetilde{G}(\varepsilon)}\right\rangle \mid x \in X\right\}$,

$$
\begin{aligned}
G_{\alpha}(\varepsilon)= & f_{\alpha}(\widetilde{G}(\varepsilon)) \\
= & f_{\alpha}\left(\left\{\left\langle x, \mu_{\widetilde{G}(\varepsilon)}(x), v_{\widetilde{G}(\varepsilon)}(x)\right\rangle \mid x \in X\right\}\right) \\
= & \left\{\left\langlex, \mu_{G_{\alpha}(\varepsilon)}(x)\right.\right. \\
& \left.\left.=r_{G_{\alpha}(\varepsilon)}(x) \cdot e^{i 2 \pi \omega_{G_{\alpha}(\varepsilon)}^{r}(x)}\right\rangle \mid x \in X\right\}, \quad \forall \varepsilon \in E .
\end{aligned}
$$

Here

$$
\begin{aligned}
& r_{G_{\alpha}(\varepsilon)}(x)=\left(r_{\widetilde{G}(\varepsilon)}(x)+\alpha \cdot\left(1-r_{\widetilde{G}(\varepsilon)}(x)-k_{\widetilde{G}(\varepsilon)}(x)\right)\right) \\
& \omega_{G_{\alpha}(\varepsilon)}^{r}(x) \\
& =\left[\left(\frac{\omega_{\widetilde{G}(\varepsilon)}^{r}(x)}{2 \pi}\right)+\alpha \cdot\left(1-\frac{\omega_{\widetilde{G}(\varepsilon)}^{r}(x)+\omega_{\widetilde{G}(\varepsilon)}^{k}(x)}{2 \pi}\right)\right] .
\end{aligned}
$$

In the following, we have to prove that $\mu_{F_{\alpha}(\varepsilon)}(x) \leq$ $\mu_{G_{\alpha}(\varepsilon)}(x)$; that is, $r_{F_{\alpha}(\varepsilon)}(x) \leq r_{G_{\alpha}(\varepsilon)}(x)$ and $\omega_{F_{\alpha}(\varepsilon)}^{r}(x) \leq$ $\omega_{G_{\alpha}(\varepsilon)}^{r}(x), \forall x \in X$ and $\varepsilon \in E$. Since $\xi \subseteq \tilde{\xi}$, therefore $\mu_{\widetilde{F}(\varepsilon)}(x) \leq \mu_{\widetilde{G}(\varepsilon)}(x)$ and $\nu_{\widetilde{F}(\varepsilon)}(x) \geq \nu_{\widetilde{G}(\varepsilon)}(x)$; that is, $r_{\widetilde{F}(\varepsilon)}(x) \leq r_{\widetilde{G}(\varepsilon)}(x)$ and $k_{\widetilde{F}(\varepsilon)}(x) \geq k_{\widetilde{G}(\varepsilon)}(x)$, for the amplitude terms and for the phase terms $\omega_{\widetilde{F}(\varepsilon)}^{r}(x) \leq$ $\omega_{\widetilde{G}(\varepsilon)}^{r}(x)$ and $\omega_{\widetilde{F}(\varepsilon)}^{k}(x) \geq \omega_{\widetilde{G}(\varepsilon)}^{k}(x), \forall x \in X$ and $\varepsilon \in E$. Thus, $(1-\alpha) \cdot r_{\widetilde{F}(\varepsilon)}(x) \leq(1-\alpha) \cdot r_{\widetilde{G}(\varepsilon)}(x), \alpha \cdot k_{\widetilde{F}(\varepsilon)}(x) \geq$ $\alpha \cdot k_{\widetilde{G}(\varepsilon)}(x)$, and $((1-\alpha) / 2 \pi) \cdot \omega_{\widetilde{F}(\varepsilon)}^{r}(x) \leq((1-\alpha) / 2 \pi)$. $\omega_{\widetilde{G}(\varepsilon)}^{r}(x),(\alpha / 2 \pi) \cdot \omega_{\widetilde{F}(\varepsilon)}^{k}(x) \geq(\alpha / 2 \pi) \cdot \omega_{\widetilde{G}(\varepsilon)}^{k}(x)$,

which implies $\alpha+(1-\alpha) \cdot r_{\widetilde{F}(\varepsilon)}(x)-\alpha \cdot k_{\widetilde{G}(\varepsilon)}(x) \leq$ $\alpha+(1-\alpha) \cdot r_{\widetilde{G}(\varepsilon)}(x)-\alpha \cdot k_{\widetilde{F}(\varepsilon)}(x)$ and

$$
\begin{aligned}
\alpha+ & {\left[\frac{(1-\alpha)}{2 \pi} \cdot \omega_{\widetilde{F}(\varepsilon)}^{r}(x)-\frac{\alpha}{2 \pi} \cdot \omega_{\widetilde{G}(\varepsilon)}^{k}(x)\right] } \\
& \leq \alpha+\left[\frac{(1-\alpha)}{2 \pi} \cdot \omega_{\widetilde{G}(\varepsilon)}^{r}(x)-\frac{\alpha}{2 \pi} \cdot \omega_{\widetilde{F}(\varepsilon)}^{k}(x)\right],
\end{aligned}
$$

$\Rightarrow r_{F_{\alpha}(\varepsilon)}(x) \leq r_{G_{\alpha}(\varepsilon)}(x)$ and $\omega_{F_{\alpha}(\varepsilon)}^{r}(x) \leq \omega_{G_{\alpha}(\varepsilon)}^{r}(x)$. Thus, we have $\mu_{F_{\alpha}(\varepsilon)}(x) \leq \mu_{G_{\alpha}(\varepsilon)}(x)$.

Hence, $f_{\alpha}(\xi) \subseteq f_{\alpha}(\widetilde{\xi})$.

(3) $f_{\alpha}\left(f_{\beta}(\xi)\right)=f_{\alpha}\left(\left\langle F_{\beta}, E\right\rangle\right)=\left\langle\left(F_{\beta}\right)_{\alpha}, E\right\rangle$, where $\left(F_{\beta}\right)_{\alpha}(\varepsilon)=f_{\alpha}\left(F_{\beta}(\varepsilon)\right)=f_{\alpha}\left(f_{\beta}(\widetilde{F}(\varepsilon))\right), \forall \varepsilon \in E$.

Next, we have to prove that $f_{\alpha}\left(f_{\beta}(\widetilde{F}(\varepsilon))\right)=\left(f_{\beta}(\widetilde{F}(\varepsilon))\right.$. We have

$$
\begin{aligned}
f_{\beta}(\widetilde{F}(\varepsilon)) & =f_{\beta}\left(\left\{\left\langle x, \mu_{\widetilde{F}(\varepsilon)}(x), \nu_{\widetilde{F}(\varepsilon)}(x)\right\rangle \mid x \in X\right\}\right) \\
& =\left\{\left\langle x, r_{F_{\beta}(\varepsilon)}(x) \cdot e^{i 2 \pi \omega_{F_{\beta}(\varepsilon)}^{r}(x)}\right\rangle \mid x \in X\right\}, \quad \forall \varepsilon \in E .
\end{aligned}
$$

Here

$$
\begin{aligned}
& r_{F_{\beta}(\varepsilon)}(x)=\left(r_{\widetilde{F}(\varepsilon)}(x)+\beta \cdot\left(1-r_{\widetilde{F}(\varepsilon)}(x)-k_{\widetilde{F}(\varepsilon)}(x)\right)\right) \\
& \omega_{F_{\beta}(\varepsilon)}^{r}(x) \\
& \quad=\left[\left(\frac{\omega_{\widetilde{F}(\varepsilon)}^{r}(x)}{2 \pi}\right)+\beta \cdot\left(1-\frac{\omega_{\widetilde{F}(\varepsilon)}^{r}(x)+\omega_{\widetilde{F}(\varepsilon)}^{k}(x)}{2 \pi}\right)\right] .
\end{aligned}
$$

Thus

$$
\begin{aligned}
f_{\alpha} & \left(f_{\beta}(\widetilde{F}(\varepsilon))\right) \\
& =f_{\alpha}\left(\left\{\left\langle x, r_{F_{\beta}(\varepsilon)}(x) \cdot e^{i 2 \pi \omega_{F_{\beta}(\varepsilon)}^{r}(x)}\right\rangle \mid x \in X\right\}\right) \\
& =\left\{\left\langle x, r_{F_{\beta}(\varepsilon)}(x) \cdot e^{i 2 \pi \omega_{F_{\beta}(\varepsilon)}^{r}(x)}\right\rangle \mid x \in X\right\}, \quad \forall \varepsilon \in E .
\end{aligned}
$$

From (26) and (28), we have $f_{\alpha}\left(f_{\beta}(\xi)\right)=f_{\beta}(\xi)$.

(4) Let $\xi^{c}=\langle\widetilde{F}, E\rangle^{c}=\left\langle\widetilde{F}^{c}, \neg E\right\rangle$, where $\widetilde{F}^{c}(\varepsilon)=\{\langle x$, $\left.\left.\nu_{\widetilde{F}(\neg \varepsilon)}(x), \mu_{\widetilde{F}(\neg \varepsilon)}(x)\right\rangle \mid x \in X\right\}, \forall \varepsilon \in E$. 
Then we have $f_{\alpha}\left(\xi^{c}\right)=f_{\alpha}\left(\left\langle\widetilde{F}^{c}, \neg E\right\rangle\right)=\left\langle\left(F^{c}\right)_{\alpha}, \neg E\right\rangle$, where

$$
\begin{aligned}
\left(F^{c}\right)_{\alpha}(\varepsilon)= & f_{\alpha}\left(\widetilde{F}^{c}(\varepsilon)\right) \\
= & f_{\alpha}\left(\left\{\left\langle x, v_{\widetilde{F}(\neg \varepsilon)}(x), \mu_{\widetilde{F}(\neg \varepsilon)}(x)\right\rangle \mid x \in X\right\}\right) \\
= & \left\{\left\langlex,\left(1-r_{F_{\alpha}(\neg \varepsilon)}(x)\right)\right.\right. \\
& \left.\left.\cdot \cdot e^{i 2 \pi \bar{\omega}_{\left.F_{\alpha}(\neg)\right)}^{r}(x)}\right\rangle \mid x \in X\right\}, \quad \forall \varepsilon \in \neg E .
\end{aligned}
$$

Here

$$
\begin{aligned}
1 & -r_{F_{\alpha}(\neg \varepsilon)}(x) \\
& =\left(k_{\widetilde{F}(\neg \varepsilon)}(x)+\alpha \cdot\left(1-r_{\widetilde{F}(\neg \varepsilon)}(x)-k_{\widetilde{F}(\neg \varepsilon)}(x)\right)\right) \\
\bar{\omega}_{F_{\alpha}(\neg \varepsilon)}^{r}(x) & \\
& =\left[\left(\frac{\omega_{\widetilde{F}(\neg \varepsilon)}^{k}(x)}{2 \pi}\right)+\alpha \cdot\left(1-\frac{\omega_{\widetilde{F}(\neg \varepsilon)}^{r}(x)+\omega_{\widetilde{F}(\neg \varepsilon)}^{k}(x)}{2 \pi}\right)\right] .
\end{aligned}
$$

Thus, we have $\left(f_{\alpha}\left(\xi^{c}\right)\right)^{c}=\left\langle\left(F^{c}\right)_{\alpha}, \neg E\right\rangle^{c}=$ $\left\langle\left(\left(F^{c}\right)_{\alpha}\right)^{c}, \neg \neg E\right\rangle=\left\langle\left(\left(F^{c}\right)_{\alpha}\right)^{c}, E\right\rangle, \forall \varepsilon \in E$. Consider

$$
\begin{aligned}
& \left(\left(F^{c}\right)_{\alpha}\right)^{c}(\varepsilon) \\
& \quad=\left(\left\{\left\langle x,\left(1-r_{F_{\alpha}(\neg \varepsilon)}(x)\right) \cdot e^{i 2 \pi \bar{\omega}_{F_{\alpha}(\neg \varepsilon)}^{r}(x)}\right\rangle \mid x \in X\right\}\right)^{c} \\
& \quad=\left\{\left\langle x, r_{F_{\alpha}(\neg \neg \varepsilon)}(x) \cdot e^{i 2 \pi \omega_{\left.F_{\alpha}(\neg\urcorner \varepsilon\right)}^{r}(x)}\right\rangle \mid x \in X\right\}, \quad \forall \varepsilon \in \neg \neg E, \\
& \quad=\left\{\left\langle x, r_{F_{\alpha}(\varepsilon)}(x) \cdot e^{i 2 \pi \omega_{F_{\alpha}(\varepsilon)}^{r}(x)}\right\rangle \mid x \in X\right\}, \quad \forall \varepsilon \in E .
\end{aligned}
$$

Here

$$
\begin{aligned}
& r_{F_{\alpha}(\varepsilon)}(x)=\left(1-k_{\widetilde{F}(\varepsilon)}(x)-\alpha \cdot\left(1-r_{\widetilde{F}(\varepsilon)}(x)-k_{\widetilde{F}(\varepsilon)}(x)\right)\right) ; \\
& \omega_{F_{\alpha}(\varepsilon)}^{r}(x) \\
& \quad=1-\left[\left(\frac{\omega_{\widetilde{F}(\varepsilon)}^{k}(x)}{2 \pi}\right)+\alpha \cdot\left(1-\frac{\omega_{\widetilde{F}(\varepsilon)}^{r}(x)+\omega_{\widetilde{F}(\varepsilon)}^{k}(x)}{2 \pi}\right)\right] .
\end{aligned}
$$

Since $f_{1-\alpha}(\xi)=f_{1-\alpha}(\langle\widetilde{F}, E\rangle)=\left\langle F_{1-\alpha}, E\right\rangle$, where

$$
\begin{aligned}
F_{1-\alpha}(\varepsilon) & \\
& =f_{1-\alpha}(\widetilde{F}(\varepsilon)) \\
& =f_{1-\alpha}\left(\left\{\left\langle x, \mu_{\widetilde{F}(\varepsilon)}(x), \nu_{\widetilde{F}(\varepsilon)}(x)\right\rangle \mid x \in X\right\}\right) \\
& =\left\{\left\langle x, r_{F_{1-\alpha}(\varepsilon)}(x) \cdot e^{i \omega_{F_{1-\alpha}(\varepsilon)}^{r}(x)}\right\rangle \mid x \in X\right\}, \quad \forall \varepsilon \in E,
\end{aligned}
$$

Here

$$
\begin{aligned}
r_{F_{1-\alpha}(\varepsilon)}(x)= & \left(1-k_{\widetilde{F}(\varepsilon)}(x)-\alpha \cdot\left(1-r_{\widetilde{F}(\varepsilon)}(x)-k_{\widetilde{F}(\varepsilon)}(x)\right)\right) \\
\omega_{F_{1-\alpha}(\varepsilon)}^{r}(x) & \\
= & {\left[\left(\frac{\omega_{\widetilde{F}(\varepsilon)}^{r}(x)}{2 \pi}\right)\right.} \\
& \left.+(1-\alpha) \cdot\left(1-\frac{\omega_{\widetilde{F}(\varepsilon)}^{r}(x)+\omega_{\widetilde{F}(\varepsilon)}^{k}(x)}{2 \pi}\right)\right] \\
=1- & {\left[\left(\frac{\omega_{\widetilde{F}(\varepsilon)}^{k}(x)}{2 \pi}\right)\right.} \\
& \left.+\alpha \cdot\left(1-\frac{\omega_{\widetilde{F}(\varepsilon)}^{r}(x)+\omega_{\widetilde{F}(\varepsilon)}^{k}(x)}{2 \pi}\right)\right] .
\end{aligned}
$$

Thus, $\left(\left(F^{c}\right)_{\alpha}\right)^{c}(\varepsilon)=F_{1-\alpha}(\varepsilon)$. Consequently, $\left(f_{\alpha}\left(\xi^{c}\right)\right)^{c}=$ $f_{1-\alpha}(\xi)$.

\section{Distance Measures and Entropies of Complex Intuitionistic Fuzzy Soft Set}

Based on various well-known distance functions, we introduce some distance measures between complex intuitionistic fuzzy soft sets and propose a general way to find the entropies of complex intuitionistic fuzzy soft set. We also give the structure of intuitionistic entropy of complex intuitionistic fuzzy soft sets by extending the structure of intuitionistic entropy on intuitionistic fuzzy soft sets [21].

4.1. Distance Measures between Complex Intuitionistic Fuzzy Soft Sets. The axiomatic definition of the distance measure between complex intuitionistic fuzzy soft sets has been reframed and proposed as follows.

Definition 13. Suppose that $\xi=\langle\widetilde{F}, E\rangle$ and $\widetilde{\xi}=\langle\widetilde{G}, E\rangle$ are two complex intuitionistic fuzzy soft sets over the universal set $X$. A real valued nonnegative function

$$
d: \mathscr{C} \mathscr{F} \mathscr{F} \mathcal{S} \mathcal{S}(X) \times \mathscr{C} \mathscr{F} \mathscr{F} \mathcal{S} \mathcal{S}(X) \longrightarrow[0,1]
$$

is called distance measure $\mathscr{C} \mathscr{F} \mathscr{F} \mathcal{S} \mathcal{S}(X)$, if $d$ satisfies the following properties:
$(P 1) 0 \leq d(\xi, \widetilde{\xi}) \leq 1$;
(P2) $d(\xi, \widetilde{\xi})=d(\widetilde{\xi}, \xi)$;
(P3) $d(\xi, \widetilde{\xi})=0$ if and only if $\xi=\widetilde{\xi}$;
(P4) for any $\eta=\langle\widetilde{H}, E\rangle=\left[c_{i j}\right]_{m \times n} \in \mathscr{C} \mathscr{F} \mathscr{F} \mathcal{S} \mathcal{S}(X)$, $d(\xi, \widetilde{\xi})+d(\widetilde{\xi}, \eta) \geq d(\xi, \eta)$. 
We extend and propose various distance measures for two CIFSSs as follows.

(i) Hamming distance:

$$
\begin{aligned}
d_{h}(\xi, \widetilde{\xi})=\frac{1}{4} \sum_{j=1}^{n} \sum_{i=1}^{m}[ & \left|r_{\widetilde{F}\left(\varepsilon_{j}\right)}\left(x_{i}\right)-r_{\widetilde{G}\left(\varepsilon_{j}\right)}\left(x_{i}\right)\right| \\
& +\left|k_{\widetilde{F}\left(\varepsilon_{j}\right)}\left(x_{i}\right)-k_{\widetilde{G}\left(\varepsilon_{j}\right)}\left(x_{i}\right)\right| \\
& +\frac{1}{2 \pi}\left(\left|\omega_{\widetilde{F}\left(\varepsilon_{j}\right)}^{r}\left(x_{i}\right)-\omega_{\widetilde{G}\left(\varepsilon_{j}\right)}^{r}\left(x_{i}\right)\right|\right. \\
& \left.\left.+\left|\omega_{\widetilde{F}\left(\varepsilon_{j}\right)}^{k}\left(x_{i}\right)-\omega_{\widetilde{G}\left(\varepsilon_{j}\right)}^{k}\left(x_{i}\right)\right|\right)\right] .
\end{aligned}
$$

(ii) Normalized Hamming distance:

$$
\begin{aligned}
d_{h}^{n}(\xi, \widetilde{\xi})=\frac{1}{4 m n} \sum_{j=1}^{n} \sum_{i=1}^{m}[ & \left|r_{\widetilde{F}\left(\varepsilon_{j}\right)}\left(x_{i}\right)-r_{\widetilde{G}\left(\varepsilon_{j}\right)}\left(x_{i}\right)\right| \\
& +\left|k_{\widetilde{F}\left(\varepsilon_{j}\right)}\left(x_{i}\right)-k_{\widetilde{G}\left(\varepsilon_{j}\right)}\left(x_{i}\right)\right| \\
& +\frac{1}{2 \pi}\left(\left|\omega_{\widetilde{F}\left(\varepsilon_{j}\right)}^{r}\left(x_{i}\right)-\omega_{\widetilde{G}\left(\varepsilon_{j}\right)}^{r}\left(x_{i}\right)\right|\right. \\
& \left.\left.+\left|\omega_{\widetilde{F}\left(\varepsilon_{j}\right)}^{k}\left(x_{i}\right)-\omega_{\widetilde{G}\left(\varepsilon_{j}\right)}^{k}\left(x_{i}\right)\right|\right)\right] .
\end{aligned}
$$

(iii) Euclidean distance:

$$
\begin{aligned}
d_{e}^{n}(\xi, \widetilde{\xi})^{2} & \\
=\frac{1}{4} \sum_{j=1}^{n} \sum_{i=1}^{m}[ & \left(r_{\widetilde{F}\left(e_{j}\right)}\left(x_{i}\right)-r_{\widetilde{G}\left(\varepsilon_{j}\right)}\left(x_{i}\right)\right)^{2} \\
& +\left(k_{\widetilde{F}\left(\varepsilon_{j}\right)}\left(x_{i}\right)-k_{\widetilde{G}\left(\varepsilon_{j}\right)}\left(x_{i}\right)\right)^{2} \\
& +\frac{1}{4 \pi^{2}}\left(\left(\omega_{\widetilde{F}\left(\varepsilon_{j}\right)}^{r}\left(x_{i}\right)-\omega_{\widetilde{G}\left(\varepsilon_{j}\right)}^{r}\left(x_{i}\right)\right)^{2}\right. \\
& \left.\left.+\left(\omega_{\widetilde{F}\left(\varepsilon_{j}\right)}^{k}\left(x_{i}\right)-\omega_{\widetilde{G}\left(\varepsilon_{j}\right)}^{k}\left(x_{i}\right)\right)^{2}\right)\right] .
\end{aligned}
$$

(iv) Normalized Euclidean distance:

$$
\begin{aligned}
d_{e}^{n}(\xi, \widetilde{\xi})^{2} & \\
=\frac{1}{4 m n} \sum_{j=1}^{n} \sum_{i=1}^{m}[ & \left(r_{\widetilde{F}\left(e_{j}\right)}\left(x_{i}\right)-r_{\widetilde{G}\left(\varepsilon_{j}\right)}\left(x_{i}\right)\right)^{2} \\
& +\left(k_{\widetilde{F}\left(\varepsilon_{j}\right)}\left(x_{i}\right)-k_{\widetilde{G}\left(\varepsilon_{j}\right)}\left(x_{i}\right)\right)^{2} \\
& +\frac{1}{4 \pi^{2}}\left(\left(\omega_{\widetilde{F}\left(\varepsilon_{j}\right)}^{r}\left(x_{i}\right)-\omega_{\widetilde{G}\left(\varepsilon_{j}\right)}^{r}\left(x_{i}\right)\right)^{2}\right. \\
& \left.\left.+\left(\omega_{\widetilde{F}\left(\varepsilon_{j}\right)}^{k}\left(x_{i}\right)-\omega_{\widetilde{G}\left(\varepsilon_{j}\right)}^{k}\left(x_{i}\right)\right)^{2}\right)\right] .
\end{aligned}
$$

4.2. Entropies on Complex Intuitionistic Fuzzy Soft Sets. Here, we present the axiomatic definition for the entropy of complex intuitionistic fuzzy soft sets. The following conditions give the intuitive idea for the degree of fuzziness of a complex intuitionistic fuzzy soft set, that is, for the entropy of a complex intuitionistic fuzzy soft set.

(i) It will be null when the complex intuitionistic fuzzy soft set is a complex fuzzy soft set.

(ii) It will be maximum if the complex intuitionistic fuzzy soft set is completely intuitionistic.

(iii) An intuitionistic entropy of a complex intuitionistic fuzzy soft set will be equal to its complement.

(iv) If the degree of membership and the degree of nonmembership of each element increase, the sum will do so as well, and therefore this complex intuitionistic fuzzy soft set becomes less fuzzy, and therefore the entropy should decrease.

In view of the above-stated points and the definition of entropy for an intuitionistic fuzzy soft set given in [21], we propose the following definition for the entropy of a complex intuitionistic fuzzy soft set.

Definition 14. A real valued function $H: \mathscr{C} \mathscr{F} \mathscr{F} \mathcal{S} \mathcal{S} \rightarrow \mathbb{R}^{+}$ is called an intuitionistic entropy on $\mathscr{C} \mathscr{I} \mathscr{F} \mathcal{S} \mathcal{S}(X)$, if $H$ has the following properties:

(i) P1 (sharpness): $H(\xi)=0$ if and only if $\xi$ is a complex fuzzy soft set;

(ii) P2 (maximality): let $\xi=\langle\widetilde{F}, E\rangle=\left[a_{i j}\right]_{m \times n}, H(\xi)=m n$ if and only if $\mu_{\widetilde{F}(\varepsilon)}(x)=\nu_{\widetilde{F}(\varepsilon)}(x)=0, \forall x \in X$ and $\varepsilon \in E$;

(iii) P3 (symmetry): $H(\xi)=H\left(\xi^{c}\right)$, for all $\xi \epsilon$ $\mathscr{C} \mathscr{I} \mathscr{F} S \mathcal{S}(X)$

(iv) P4 (resolution): if $\xi^{*}$ is sharpened version of $\xi$, that is, if $\xi \preceq \xi^{*}$, then $H(\xi) \geq H\left(\xi^{*}\right)$.

Similar to the entropy of an intuitionistic fuzzy soft set [21], we deduce the following property from the property P2 of Definition 14.

Theorem 15. $H(\xi)$ is maximum if and only if $\xi=\langle\widetilde{F}, E\rangle=$ $\left[a_{i j}\right]_{m \times n}=[0]_{m \times n}$; that is, $a_{i j}=\mu_{\widetilde{F}\left(\varepsilon_{j}\right)}\left(x_{i}\right)=\nu_{\widetilde{F}\left(\varepsilon_{j}\right)}\left(x_{i}\right)=0$, $\forall \varepsilon_{j} \in E, x_{i} \in X$, where $0 \leq i \leq m$ and $0 \leq j \leq n$.

Proof. Consider the following.

Necessary Part. Let $\xi=\langle\widetilde{F}, E\rangle=\left[a_{i j}\right]_{m \times n}=[0]_{m \times n}$. Let $\widetilde{\xi}=\langle\widetilde{G}, E\rangle$ be any complex intuitionistic fuzzy soft set. Since $\mu_{\widetilde{G}\left(\varepsilon_{j}\right)}\left(x_{i}\right) \geq 0$ and $\nu_{\widetilde{G}\left(\varepsilon_{j}\right)}\left(x_{i}\right) \geq 0, \forall \varepsilon_{j} \in E, x_{i} \in X$, where $0 \leq i \leq m$ and $0 \leq j \leq n$, therefore, by Definition 10, we have $\xi \preceq \widetilde{\xi}$. Thus, $H(\xi) \geq H(\widetilde{\xi})$ by property $\mathrm{P} 4$ of Definition 14 for all $\tilde{\xi}$, and then $H(\xi)$ is maximum.

Sufficient Part. Let $H(\xi)$ is maximum. We assume that $\xi=$ $\langle\widetilde{F}, E\rangle=\left[a_{i j}\right]_{m \times n} \neq[0]_{m \times n}$, and then there is a $\varepsilon_{j} \in E$ and 
$x_{i} \in X$ such that $\mu_{\widetilde{F}\left(\varepsilon_{j}\right)}\left(x_{i}\right) \neq 0$ and $\nu_{\widetilde{F}\left(\varepsilon_{j}\right)}\left(x_{i}\right) \neq 0$, where $0 \leq i \leq m$ and $0 \leq j \leq n$. We construct the following complex intuitionistic fuzzy soft set: $\widetilde{\xi}=\langle\widetilde{G}, E\rangle$ with $\mu_{\widetilde{G}\left(\varepsilon_{i}\right)}\left(x_{i}\right)=$ $(1 / 2) \mu_{\widetilde{F}\left(\varepsilon_{j}\right)}\left(x_{i}\right)$ and $\nu_{\widetilde{G}\left(\varepsilon_{j}\right)}\left(x_{i}\right)=(1 / 3) \nu_{\widetilde{F}\left(\varepsilon_{j}\right)}\left(x_{i}\right)$ for all $\varepsilon_{j} \in E$ and $x_{i} \in X$, and then by Definition 10 we have $H(\widetilde{\xi}) \geq H(\xi)$ which contradicts the fact that hypothesis $H(\xi)$ is maximum. Therefore, $\xi=[0]_{m \times n}$.

Let us take the following set $D=\{(x, y) \in[0,1] \times[0,1]$ | $x+y \leq 1\}$ and with it let us construct $\psi_{D}: D \rightarrow[0,1]$, which satisfies the following conditions:

(1) $\psi_{D}(x, y)=1$ if and only if $x+y=1$;

(2) $\psi_{D}(x, y)=0$ if and only if $x=0=y$;

(3) $\psi_{D}(x, y)=\psi_{D}(y, x)$;

(4) if $x \leq x^{\prime}$ and $y \leq y^{\prime}$, then $\psi_{D}(x, y) \leq \psi_{D}\left(x^{\prime}, y^{\prime}\right)$.

Theorem 16. Let $\xi=\langle\widetilde{F}, E\rangle=\left[a_{i j}\right]_{m \times n} \in \mathscr{C} \mathscr{F} \mathscr{F} \mathcal{S} \mathcal{S}(X)$ and $H: \mathscr{C} \mathscr{J} \mathscr{F} \mathcal{S} \mathcal{S}(X) \rightarrow \mathbb{R}^{+}$such that

$$
\begin{aligned}
H(\xi)=\frac{1}{2} \sum_{j=1}^{n} \sum_{i=1}^{m}[ & \left(1-\psi\left(r_{\widetilde{F}\left(\varepsilon_{j}\right)}\left(x_{i}\right), k_{\widetilde{F}\left(\varepsilon_{j}\right)}\left(x_{i}\right)\right)\right) \\
& \left.+\left(1-\psi\left(\frac{\omega_{\widetilde{F}\left(\varepsilon_{j}\right)}^{r}\left(x_{i}\right)}{2 \pi}, \frac{\omega_{\widetilde{F}\left(\varepsilon_{j}\right)}^{k}\left(x_{i}\right)}{2 \pi}\right)\right)\right],
\end{aligned}
$$

where $\psi$ satisfies conditions (1)-(4); then $H$ is an intuitionistic entropy on $\mathscr{C} \mathscr{F} \mathscr{F} \mathcal{S} \mathcal{S}(X)$.

Proof. (1) $H(\xi)=0$ if and only if, for all $\varepsilon_{j} \in E, x_{i} \in X$,

$$
\begin{aligned}
\frac{1}{2} \sum_{j=1}^{n} \sum_{i=1}^{m}[ & \left(1-\psi\left(r_{\widetilde{F}\left(\varepsilon_{j}\right)}\left(x_{i}\right), k_{\widetilde{F}\left(\varepsilon_{j}\right)}\left(x_{i}\right)\right)\right) \\
& \left.+\left(1-\psi\left(\frac{\omega_{\widetilde{F}\left(\varepsilon_{j}\right)}^{r}\left(x_{i}\right)}{2 \pi}, \frac{\omega_{\widetilde{F}\left(\varepsilon_{j}\right)}^{k}\left(x_{i}\right)}{2 \pi}\right)\right)\right]=0, \\
\Longleftrightarrow & \psi\left(r_{\widetilde{F}\left(\varepsilon_{j}\right)}\left(x_{i}\right), k_{\widetilde{F}\left(\varepsilon_{j}\right)}\left(x_{i}\right)\right)=1, \\
& \psi\left(\frac{\omega_{\widetilde{F}\left(\varepsilon_{j}\right)}^{r}\left(x_{i}\right)}{2 \pi}, \frac{\omega_{\widetilde{F}\left(\varepsilon_{j}\right)}^{k}\left(x_{i}\right)}{2 \pi}\right)=1, \\
\Longleftrightarrow & r_{\widetilde{F}\left(\varepsilon_{j}\right)}\left(x_{i}\right)+k_{\widetilde{F}\left(\varepsilon_{j}\right)}\left(x_{i}\right)=1, \\
& \omega_{\widetilde{F}\left(\varepsilon_{j}\right)}^{r}\left(x_{i}\right)+\omega_{\widetilde{F}\left(\varepsilon_{j}\right)}^{k}\left(x_{i}\right)=2 \pi, \\
\Longleftrightarrow & \xi \in C F S S(X) .
\end{aligned}
$$

Thus, $H$ satisfies property P1 of Definition 14.
(2) $H(\xi)=m n$ if and only if, for all $\varepsilon_{j} \in E, x_{i} \in X$,

$$
\begin{aligned}
& \frac{1}{2} \sum_{j=1}^{n} \sum_{i=1}^{m}\left[\left(1-\psi\left(r_{\widetilde{F}\left(\varepsilon_{j}\right)}\left(x_{i}\right), k_{\widetilde{F}\left(\varepsilon_{j}\right)}\left(x_{i}\right)\right)\right)\right. \\
& \left.\quad+\left(1-\psi\left(\frac{\omega_{\widetilde{F}\left(\varepsilon_{j}\right)}^{r}\left(x_{i}\right)}{2 \pi}, \frac{\omega_{\widetilde{F}\left(\varepsilon_{j}\right)}^{k}\left(x_{i}\right)}{2 \pi}\right)\right)\right]=m n, \\
& \Longleftrightarrow r_{\widetilde{F}\left(\varepsilon_{j}\right)}\left(x_{i}\right)+k_{\widetilde{F}\left(\varepsilon_{j}\right)}\left(x_{i}\right)=0, \\
& \quad \omega_{\widetilde{F}\left(\varepsilon_{j}\right)}^{r}\left(x_{i}\right)+\omega_{\widetilde{F}\left(\varepsilon_{j}\right)}^{k}\left(x_{i}\right)=0, \\
& \Longleftrightarrow r_{\widetilde{F}\left(\varepsilon_{j}\right)}\left(x_{i}\right), k_{\widetilde{F}\left(\varepsilon_{j}\right)}\left(x_{i}\right)=0, \\
& \quad \omega_{\widetilde{F}\left(\varepsilon_{j}\right)}^{r}\left(x_{i}\right), \omega_{\widetilde{F}\left(\varepsilon_{j}\right)}^{k}\left(x_{i}\right)=0,
\end{aligned}
$$

which implies $\xi$ is completely intuitionistic. Thus, $H$ satisfies property $\mathrm{P} 2$ of Definition 14.

(3) Since $\xi^{c}=\langle\widetilde{F}, E\rangle^{c}=\left\langle\widetilde{F}^{c}, \neg E\right\rangle$, where $\widetilde{F}^{c}=$ $\left\{\left\langle x_{i}, v_{\widetilde{F}(\varepsilon)}\left(x_{i}\right), \mu_{\widetilde{F}(\varepsilon)}\left(x_{i}\right)\right\rangle \mid x_{i} \in X\right\}, \forall \neg \varepsilon \in \neg E$, then

$$
\begin{aligned}
H(\xi)=\frac{1}{2} \sum_{j=1}^{n} \sum_{i=1}^{m}\left[\left(1-\psi\left(r_{\widetilde{F}\left(\varepsilon_{j}\right)}\left(x_{i}\right), k_{\widetilde{F}\left(\varepsilon_{j}\right)}\left(x_{i}\right)\right)\right)\right. \\
\left.+\left(1-\psi\left(\frac{\omega_{\widetilde{F}\left(\varepsilon_{j}\right)}^{r}\left(x_{i}\right)}{2 \pi}, \frac{\omega_{\widetilde{F}\left(\varepsilon_{j}\right)}^{k}\left(x_{i}\right)}{2 \pi}\right)\right)\right] \\
=\frac{1}{2} \sum_{j=1}^{n} \sum_{i=1}^{m}\left[\left(1-\psi\left(k_{\widetilde{F}\left(\varepsilon_{j}\right)}\left(x_{i}\right), r_{\widetilde{F}\left(\varepsilon_{j}\right)}\left(x_{i}\right)\right)\right)\right. \\
\left.\quad+\left(1-\psi\left(\frac{\omega_{\widetilde{F}\left(\varepsilon_{j}\right)}^{k}\left(x_{i}\right)}{2 \pi}, \frac{\omega_{\widetilde{F}\left(\varepsilon_{j}\right)}^{r}\left(x_{i}\right)}{2 \pi}\right)\right)\right] \\
=H\left(\xi^{c}\right) . \quad
\end{aligned}
$$

Thus, $H$ satisfies property P3 of Definition 14 .

(4) Let $\tilde{\xi}=\langle\widetilde{F}, E\rangle=\left[b_{i j}\right]_{m \times n}$, and if $\xi \leq \widetilde{\xi}$, then we have $r_{\widetilde{F}\left(\varepsilon_{j}\right)}\left(x_{i}\right) \leq r_{\widetilde{G}\left(\varepsilon_{j}\right)}\left(x_{i}\right)$ and $k_{\widetilde{F}\left(\varepsilon_{j}\right)}\left(x_{i}\right) \leq k_{\widetilde{G}\left(\varepsilon_{j}\right)}\left(x_{i}\right)$, for the amplitude terms and for the phase terms $\omega_{\widetilde{F}\left(\varepsilon_{j}\right)}^{r}\left(x_{i}\right) \leq$ $\omega_{\widetilde{G}\left(\varepsilon_{j}\right)}^{r}\left(x_{i}\right)$ and $\omega_{\widetilde{F}\left(\varepsilon_{j}\right)}^{k}\left(x_{i}\right) \leq \omega_{\widetilde{G}\left(\varepsilon_{j}\right)}^{k}\left(x_{i}\right), \forall x_{i} \in X$ and $\forall \varepsilon_{j} \in E$. 
Thus, we have

$$
\begin{gathered}
\psi\left(r_{\widetilde{F}\left(\varepsilon_{j}\right)}\left(x_{i}\right), k_{\widetilde{F}\left(\varepsilon_{j}\right)}\left(x_{i}\right)\right) \leq \psi\left(r_{\widetilde{G}\left(\varepsilon_{j}\right)}\left(x_{i}\right), k_{\widetilde{G}\left(\varepsilon_{j}\right)}\left(x_{i}\right)\right) \\
\psi\left(\frac{\omega_{\widetilde{F}\left(\varepsilon_{j}\right)}^{r}\left(x_{i}\right)}{2 \pi}, \frac{\omega_{\widetilde{F}\left(\varepsilon_{j}\right)}^{k}\left(x_{i}\right)}{2 \pi}\right) \leq \psi\left(\frac{\omega_{\widetilde{G}\left(\varepsilon_{j}\right)}^{r}\left(x_{i}\right)}{2 \pi}, \frac{\omega_{\widetilde{G}\left(\varepsilon_{j}\right)}^{k}\left(x_{i}\right)}{2 \pi}\right) \\
\Longrightarrow\left(1-\psi\left(r_{\widetilde{F}\left(\varepsilon_{j}\right)}\left(x_{i}\right), k_{\widetilde{F}\left(\varepsilon_{j}\right)}\left(x_{i}\right)\right)\right) \\
\geq\left(1-\psi\left(r_{\widetilde{G}\left(\varepsilon_{j}\right)}\left(x_{i}\right), k_{\widetilde{G}\left(\varepsilon_{j}\right)}\left(x_{i}\right)\right)\right), \\
\left(1-\psi\left(\frac{\omega_{\widetilde{F}\left(\varepsilon_{j}\right)}^{r}\left(x_{i}\right)}{2 \pi}, \frac{\omega_{\widetilde{F}\left(\varepsilon_{j}\right)}^{k}\left(x_{i}\right)}{2 \pi}\right)\right) \\
\geq\left(1-\psi\left(\frac{\omega_{\widetilde{G}\left(\varepsilon_{j}\right)}^{r}\left(x_{i}\right)}{2 \pi}, \frac{\omega_{\widetilde{G}\left(\varepsilon_{j}\right)}^{k}\left(x_{i}\right)}{2 \pi}\right)\right) \\
\Longrightarrow H(\tilde{\xi}) \leq H(\xi) .
\end{gathered}
$$

Thus, $H$ satisfies property P4 of Definition 14 .

Therefore, $H$ is an intuitionistic entropy of complex intuitionistic fuzzy soft set.

Burillo and Bustince [23] gave some expressions for intuitionistic entropy of intuitionistic fuzzy soft sets. Jiang et al. [21] extended these expressions for intuitionistic entropy of intuitionistic fuzzy soft sets. On similar pattern, we are extending these expressions for intuitionistic entropy of complex intuitionistic fuzzy soft sets.

Let $\xi=\langle\widetilde{F}, E\rangle=\left[a_{i j}\right]_{m \times n} \in \mathscr{C} \mathscr{F} \mathscr{F} \mathcal{S} \mathcal{S}(X)$. It is easy to verify that the following expressions are the intuitionistic entropies of $\xi$ :

$$
\begin{aligned}
H_{1}(\xi)=\frac{1}{2} \sum_{j=1}^{n} \sum_{i=1}^{m}\left[\left(1-\left(r_{\widetilde{F}\left(\varepsilon_{j}\right)}\left(x_{i}\right)+k_{\widetilde{F}\left(\varepsilon_{j}\right)}\left(x_{i}\right)\right)\right)\right. \\
\left.+\left(1-\frac{\left(\omega_{\widetilde{F}\left(\varepsilon_{j}\right)}^{r}\left(x_{i}\right)+\omega_{\widetilde{F}\left(\varepsilon_{j}\right)}^{k}\left(x_{i}\right)\right)}{2 \pi}\right)\right] ;
\end{aligned}
$$

$H_{2}(\xi)$

$$
\begin{aligned}
&=\frac{1}{2} \sum_{j=1}^{n} \sum_{i=1}^{m}\left[\left(1-\left(r_{\widetilde{F}\left(\varepsilon_{j}\right)}\left(x_{i}\right)+k_{\widetilde{F}\left(\varepsilon_{j}\right)}\left(x_{i}\right)\right)^{n}\right)\right. \\
&\left.+\left(1-\left(\frac{\left(\omega_{\widetilde{F}\left(\varepsilon_{j}\right)}^{r}\left(x_{i}\right)+\omega_{\widetilde{F}\left(\varepsilon_{j}\right)}^{k}\left(x_{i}\right)\right)}{2 \pi}\right)\right)\right], \\
& n=2,3, \ldots ;
\end{aligned}
$$

$$
\begin{aligned}
& H_{3}(\xi)=\frac{1}{2} \sum_{j=1}^{n} \sum_{i=1}^{m}\left[\left(1-\left(r_{\widetilde{F}\left(\varepsilon_{j}\right)}\left(x_{i}\right)+k_{\widetilde{F}\left(\varepsilon_{j}\right)}\left(x_{i}\right)\right)\right.\right. \\
& \left.\cdot e^{1-\left(r_{\tilde{F}\left(\xi_{j}\right)}\left(x_{i}\right)+k_{\tilde{F}\left(\xi_{j}\right)}\left(x_{i}\right)\right)}\right), \\
& +\left(1-\frac{\left(\omega_{\widetilde{F}\left(\varepsilon_{j}\right)}^{r}\left(x_{i}\right)+\omega_{\widetilde{F}\left(\varepsilon_{j}\right)}^{k}\left(x_{i}\right)\right)}{2 \pi}\right. \\
& \left.\left.\cdot e^{1-\left(\omega_{\tilde{F}\left(\xi_{j}\right)}^{r}\left(x_{i}\right)+\omega_{\tilde{F}\left(\xi_{j}\right)}^{k}\left(x_{i}\right)\right) / 2 \pi}\right)\right] \\
& H_{4}(\xi)=\frac{1}{2} \sum_{j=1}^{n} \sum_{i=1}^{m}\left[\left(1-\left(r_{\widetilde{F}\left(\varepsilon_{j}\right)}\left(x_{i}\right)+k_{\widetilde{F}\left(\varepsilon_{j}\right)}\left(x_{i}\right)\right)\right.\right. \\
& \left.\cdot \sin \left(\left(\frac{\pi}{2}\right)\left(r_{\widetilde{F}\left(\varepsilon_{j}\right)}\left(x_{i}\right)+k_{\widetilde{F}\left(\varepsilon_{j}\right)}\left(x_{i}\right)\right)\right)\right) \\
& +\left(1-\frac{\left(\omega_{\widetilde{F}\left(\varepsilon_{j}\right)}^{r}\left(x_{i}\right)+\omega_{\widetilde{F}\left(\varepsilon_{j}\right)}^{k}\left(x_{i}\right)\right)}{2 \pi}\right) \\
& \left.\cdot \sin \left(\frac{\left(\omega_{\widetilde{F}\left(\varepsilon_{j}\right)}^{r}\left(x_{i}\right)+\omega_{\widetilde{F}\left(\varepsilon_{j}\right)}^{k}\left(x_{i}\right)\right)}{4}\right)\right] .
\end{aligned}
$$

In the following definition, we introduce a function from $\mathscr{C} \mathscr{F} \mathscr{F} \mathcal{S} \mathcal{S}(X)$ to $\mathbb{R}^{+}$, which is an extension of the $H_{\phi, \phi^{\prime}}$ function from $\mathscr{I} \mathscr{F} \mathcal{S} \mathcal{S}(X)$ to $\mathbb{R}^{+}$given in [21], which is also an extension of the $H_{\phi, \phi^{\prime}}$-function from $\mathscr{F} \mathcal{S} \mathcal{S}(X)$ to $\mathbb{R}^{+}$given in [23].

Definition 17. Let $\phi, \phi^{\prime}:[0,1] \rightarrow[0,1]$ be such that if $x+$ $y \leq 1$, then $\phi(x)+\phi^{\prime}(y) \leq 1$, with $x, y \in[0,1]$. One defines function $H_{\phi, \phi^{\prime}}(\cdot)$ of the complex intuitionistic fuzzy soft set $\xi=\langle\widetilde{F}, E\rangle=\left[a_{i j}\right]_{m \times n} \in \mathscr{C} \mathscr{I} \mathscr{F} \mathcal{S} \mathcal{S}(X)$ to $\mathbb{R}^{+}$as follows:

$$
\begin{aligned}
& H_{\phi, \phi^{\prime}}(\xi) \\
& =m n-\frac{1}{2} \sum_{j=1}^{n} \sum_{i=1}^{m}\left[\left(\phi\left(r_{\widetilde{F}\left(\varepsilon_{j}\right)}\left(x_{i}\right)\right)+\phi^{\prime}\left(k_{\widetilde{F}\left(\varepsilon_{j}\right)}\left(x_{i}\right)\right)\right)\right. \\
& +\left(\phi\left(\frac{\omega_{\widetilde{F}\left(\varepsilon_{j}\right)}^{r}\left(x_{i}\right)}{2 \pi}\right)\right. \\
& \left.\left.+\phi^{\prime}\left(\frac{\omega_{\widetilde{F}\left(\varepsilon_{j}\right)}^{k}\left(x_{i}\right)}{2 \pi}\right)\right)\right] .
\end{aligned}
$$


Obviously, $0 \leq H_{\phi, \phi^{\prime}}(\xi) \leq m n$ for all $\xi=\left[a_{i j}\right]_{m \times n}$ belonging to $\mathscr{C} \mathscr{Y} \mathscr{F} \mathcal{S} \mathcal{S}(X)$.

Theorem 18. Let $\xi=\langle\widetilde{F}, F\rangle=\left[a_{i j}\right]_{m \times n}, \widetilde{\xi}=\langle\widetilde{G}, E\rangle=$ $\left[b_{i j}\right]_{m \times n} \in \mathscr{C} \mathscr{S} \mathscr{F} \mathcal{S} \mathcal{S}(X)$; then function $H_{\phi, \phi^{\prime}}$ satisfies the following property:

$$
H_{\phi, \phi^{\prime}}(\xi \cup \widetilde{\xi})+H_{\phi, \phi^{\prime}}(\xi \cap \widetilde{\xi})=H_{\phi, \phi^{\prime}}(\xi)+H_{\phi, \phi^{\prime}}(\widetilde{\xi}) .
$$

Proof. By Definition 17, we have the following:

$$
\begin{aligned}
H_{\phi, \phi^{\prime}}(\xi \cup \widetilde{\xi}) & \\
=m n-\frac{1}{2} \sum_{j=1}^{n} \sum_{i=1}^{m}[ & \left(\phi\left(r_{\widetilde{F}\left(\varepsilon_{j}\right)}\left(x_{i}\right) \diamond r_{\widetilde{G}\left(\varepsilon_{j}\right)}\left(x_{i}\right)\right)\right. \\
& \left.+\phi^{\prime}\left(k_{\widetilde{F}\left(\varepsilon_{j}\right)}\left(x_{i}\right) * k_{\widetilde{F}\left(\varepsilon_{j}\right)}\left(x_{i}\right)\right)\right) \\
& +\phi\left(\frac{\omega_{\widetilde{F}\left(\varepsilon_{j}\right)}^{r}\left(x_{i}\right)}{2 \pi} \cup \frac{\omega_{\widetilde{G}\left(\varepsilon_{j}\right)}^{r}\left(x_{i}\right)}{2 \pi}\right) \\
& \left.+\phi^{\prime}\left(\frac{\omega_{\widetilde{F}\left(\varepsilon_{j}\right)}^{k}\left(x_{i}\right)}{2 \pi} \cap \frac{\omega_{\widetilde{G}\left(\varepsilon_{j}\right)}^{k}\left(x_{i}\right)}{2 \pi}\right)\right],
\end{aligned}
$$

$$
\begin{aligned}
H_{\phi, \phi^{\prime}}(\xi \cap \tilde{\xi}) & \\
=m n-\frac{1}{2} \sum_{j=1}^{n} \sum_{i=1}^{m}[ & \left(\phi\left(r_{\widetilde{F}\left(\varepsilon_{j}\right)}\left(x_{i}\right) * r_{\widetilde{G}\left(\varepsilon_{j}\right)}\left(x_{i}\right)\right)\right. \\
& \left.+\phi^{\prime}\left(k_{\widetilde{F}\left(\varepsilon_{j}\right)}\left(x_{i}\right) \diamond k_{\widetilde{F}\left(\varepsilon_{j}\right)}\left(x_{i}\right)\right)\right) \\
& +\phi\left(\frac{\omega_{\widetilde{F}\left(\varepsilon_{j}\right)}^{r}\left(x_{i}\right)}{2 \pi} \cup \frac{\omega_{\widetilde{G}\left(\varepsilon_{j}\right)}^{r}\left(x_{i}\right)}{2 \pi}\right) \\
& \left.+\phi^{\prime}\left(\frac{\omega_{\widetilde{F}\left(\varepsilon_{j}\right)}^{k}\left(x_{i}\right)}{2 \pi} \cap \frac{\omega_{\widetilde{G}\left(\varepsilon_{j}\right)}^{k}\left(x_{i}\right)}{2 \pi}\right)\right] .
\end{aligned}
$$

Thus, we have

$$
H_{\phi, \phi^{\prime}}(\xi \cup \widetilde{\xi})+H_{\phi, \phi^{\prime}}(\xi \cap \widetilde{\xi})=H_{\phi, \phi^{\prime}}(\xi)+H_{\phi, \phi^{\prime}}(\widetilde{\xi})
$$

It may be noted that there are $H_{\phi, \phi^{\prime}}$-functions which are not intuitionistic entropies; for example,

$$
\begin{aligned}
& H_{\phi, \phi^{\prime}}(\xi) \\
& =m n-\frac{1}{2} \sum_{j=1}^{n} \sum_{i=1}^{m}\left[\left(r_{\widetilde{F}\left(\varepsilon_{j}\right)}\left(x_{i}\right)+k_{\widetilde{F}\left(\varepsilon_{j}\right)}\left(x_{i}\right)\right)\right. \\
& \left.+\frac{1}{2 \pi}\left(\omega_{\widetilde{F}\left(\varepsilon_{j}\right)}^{r}\left(x_{i}\right)+\omega_{\widetilde{F}\left(\varepsilon_{j}\right)}^{k}\left(x_{i}\right)\right)\right] .
\end{aligned}
$$

On the other hand, it may also be easily verified that there are entropies which are not $H_{\phi, \phi^{\prime}}$-functions; for example,

$$
\begin{aligned}
H_{\phi, \phi^{\prime}}(\xi) & \\
=m n-\frac{1}{2} \sum_{j=1}^{n} \sum_{i=1}^{m}[ & \left(r_{\widetilde{F}\left(\varepsilon_{j}\right)}\left(x_{i}\right)+k_{\widetilde{F}\left(\varepsilon_{j}\right)}\left(x_{i}\right)\right)^{2} \\
& \left.+\left(\frac{1}{2 \pi}\left(\omega_{\widetilde{F}\left(\varepsilon_{j}\right)}^{r}\left(x_{i}\right)+\omega_{\widetilde{F}\left(\varepsilon_{j}\right)}^{k}\left(x_{i}\right)\right)\right)^{2}\right] .
\end{aligned}
$$

Also, there are entropies which are also $H_{\phi, \phi^{\prime}}$-functions; for example,

$$
\begin{aligned}
H_{\phi, \phi^{\prime}}(\xi) & \\
=m n-\frac{1}{2} \sum_{j=1}^{n} \sum_{i=1}^{m}[ & \left(1-\left(r_{\widetilde{F}\left(\varepsilon_{j}\right)}\left(x_{i}\right)+k_{\widetilde{F}\left(\varepsilon_{j}\right)}\left(x_{i}\right)\right)\right) \\
& \left.+\left(1-\frac{1}{2 \pi}\left(\omega_{\widetilde{F}\left(\varepsilon_{j}\right)}^{r}\left(x_{i}\right)+\omega_{\widetilde{F}\left(\varepsilon_{j}\right)}^{k}\left(x_{i}\right)\right)\right)\right] .
\end{aligned}
$$

Next, we introduce a property which defines entropies in a general way as follows.

Theorem 19. If $\phi:[0,1] \rightarrow[0,1]$ satisfies the following conditions:

(1) $\phi$ is increasing,

(2) $\phi(x)=0$ if and only if $x=0$,

(3) $\phi(x)+\phi(y)=1$ if and only if $x+y=1$, then $\phi(x)+$ $\phi(y)$ satisfies conditions (1)-(4) of $\psi$ function defined previously.

We denote $H_{\phi, \phi^{\prime}}$-function as $H_{\phi, \phi^{-}}$function if $\phi=\phi^{\prime}$. The following theorem characterizes the intuitionistic entropy of complex intuitionistic fuzzy soft sets in a general way.

Theorem 20. Let $H: \mathscr{C} \mathscr{S} \mathscr{F} \mathcal{S} \mathcal{S} \rightarrow R^{+}, \phi:[0,1] \rightarrow[0,1]$, and $\xi=\langle\widetilde{F}, E\rangle=\left[a_{i j}\right]_{m \times n} \in \mathscr{C} \mathscr{F} \mathscr{F} \mathcal{S} \mathcal{S}(X)$. H is an intuitionistic entropy and $H_{\phi, \phi}$-function if and only if

$H(\xi)$

$$
\begin{aligned}
=\frac{1}{2} \sum_{j=1}^{n} \sum_{i=1}^{m}[ & \left(1-\left(\phi\left(r_{\widetilde{F}\left(\varepsilon_{j}\right)}\left(x_{i}\right)\right)+\phi\left(k_{\widetilde{F}\left(\varepsilon_{j}\right)}\left(x_{i}\right)\right)\right)\right) \\
+ & \left(1-\left(\frac{\omega_{\widetilde{F}\left(\varepsilon_{j}\right)}^{r}\left(x_{i}\right)}{2 \pi}\right)\right. \\
& \left.\left.\left.+\phi\left(\frac{\omega_{\widetilde{F}\left(\varepsilon_{j}\right)}^{k}\left(x_{i}\right)}{2 \pi}\right)\right)\right)\right],
\end{aligned}
$$

where $\phi$ satisfies conditions (1)-(3) of Theorem 19. 
Based on the definitions and properties stated above, it may be easily verified that there is a kind of correspondence between the proposed entropies and the distance measures of complex intuitionistic fuzzy soft sets, which is as follows.

Theorem 21. Let $\xi=\langle\widetilde{F}, E\rangle=\left[a_{i j}\right]_{m \times n} \in \mathscr{C} \mathscr{I F F} \mathcal{S} \mathcal{S}(X)$ and $f_{\alpha}(\xi)=\left\{\xi_{\alpha}\right\}_{\alpha \in[0,1]}$ be the family of complex fuzzy soft sets associated with $\xi$ by operator $f_{\alpha}$ defined in Definition 11. Then

(1) $H_{1}(\xi)=2 \cdot d^{h}\left(\xi, \xi_{\alpha}\right)$;

(2) $H_{1}(\xi)=2 \cdot d^{h}\left(\xi_{0}, \xi_{1}\right)$;

(3) $d^{h}\left(\xi_{\alpha}, \xi_{\beta}\right)=(\alpha-\beta) \cdot H_{1}(\xi)$

\section{Application in Multicriteria Decision Making problems}

Suppose that a car dealer $X$ decides to purchase cars from a car company $Y$. The car company provides some information to the car dealer on four different models of cars with different manufacturing dates for each model. So, the car dealer $X$ wants to select four models, say, Car1, Car2, Car3, and Car4, with its manufacturing date simultaneously. Suppose that a team of experts (decision makers) agreed that five parameters should be considered in the selection process and they can be reliability, maximum payload, purchasing cost, maximum speed, and durability. It may be noted that the parameters may get affected and altered if the manufacturing date is different for a particular model of car. The decision made by the expert team will also depend on the knowledge and experience of its members. The best way to represent this kind of information may be by using CIFSS, in which, for each car model, the experts have different opinions and mentalities. For instance, suppose that at least $60 \%$ of experts believe that Carl is suitable on the first parameter and not more than $15 \%$ of the experts believe that Carl is poor on the first parameter. In this way, we can calculate the amplitude terms for both membership and nonmembership functions of CIFSS. The phase terms that represent the manufacturing date for the first parameter of Carl can be calculated as follows: whether at least $70 \%$ of experts believe that the production date of Carl is suitable at the first parameter and not more than $20 \%$ of them believe that the production date of Carl is poor. Therefore, the information based on experts about Car1 on the first parameter can be represented in the form of CIFSS as $\left\langle 0.6 \cdot e^{2 \pi 0.70}, 0.15 \cdot e^{2 \pi 0.20}\right\rangle$. In this way, all data can be obtained in the form of CIFSS, where both amplitude and phase terms can represent the information on experts's decision which happens periodically. Assume that the expert team had suggested an ideal car (i.e., a car that is in demand) before getting the characteristic information from car maker $Y$. The aim of the expert team is to select a suitable car listed by car maker $Y$ that is most likely to be the ideal car. Using this information, car dealer $X$ can take the decision to purchase cars from car company $Y$ which are in demand in the market to gain maximum profit.

\section{Conclusions}

The new introduced concept of complex intuitionistic fuzzy soft sets (CIFSSs) which is a parametric tool has been well proposed and studied in detail along with its important properties and fundamental operations. Based on various well-known distance measures, some new distance measures for CIFSSs have been obtained and extended to find the entropies of complex intuitionistic fuzzy soft sets. A correspondence between the proposed entropies and the distance measures of complex intuitionistic fuzzy soft sets has been well established. An application in the area of multicriteria decision making problem on the basis of the proposed CIFSSs, distance measures, and information measures has also been suggested.

\section{Conflict of Interests}

The authors declare that there is no conflict of interests regarding the publication of this paper.

\section{Acknowledgments}

The authors would like to thank the anonymous reviewers for their critical comments and valuable suggestions. The general and the technical remarks of the editor and reviewers were of great help for improving the quality and presentation of the paper.

\section{References}

[1] D. Ramot, R. Milo, M. Friedman, and A. Kandel, "Complex fuzzy sets," IEEE Transactions on Fuzzy Systems, vol. 10, no. 2, pp. 171-186, 2002.

[2] D. Ramot, M. Friedman, G. Langholz, and A. Kandel, "Complex fuzzy logic," IEEE Transactions on Fuzzy Systems, vol. 11, no. 4, pp. 450-461, 2003.

[3] J. Ma, G. Zhang, and J. Lu, "A method for multiple periodic factor prediction problems using complex fuzzy sets," IEEE Transactions on Fuzzy Systems, vol. 20, no. 1, pp. 32-45, 2012.

[4] Z. Chen, S. Aghakhani, J. Man, and S. Dick, "ANCFIS: a neurofuzzy architecture employing complex fuzzy sets," IEEE Transactions on Fuzzy Systems, vol. 19, no. 2, pp. 305-322, 2011.

[5] K. T. Atanassov, "Intuitionistic fuzzy sets," Fuzzy Sets and Systems, vol. 20, no. 1, pp. 87-96, 1986.

[6] K. T. Atanassov, "More on intuitionistic fuzzy sets," Fuzzy Sets and Systems, vol. 33, no. 1, pp. 37-45, 1989.

[7] K. T. Atanassov, Intuitionistic Fuzzy Sets: Theory and Applications, Physica, Heidelberg, Germany, 1999.

[8] E. Szmidt and J. Kacprzyk, "Group decision making under Intuitionistic fuzzy preference relations," in Proceedings of 7 th Information Processing and Management of Uncertainty in Knowledge-Based Systems Conference, pp. 172-178, Paris, France, 1998.

[9] S. K. de, R. Biswas, and A. R. Roy, "An application of intuitionistic fuzzy sets in medical diagnosis," Fuzzy Sets and Systems, vol. 117, no. 2, pp. 209-213, 2001.

[10] A. Alkouri and A. Salleh, "Complex intuitionistic fuzzy sets," in Proceedings of the International Conference on Fundamental 
and Applied Sciences (ICFAS '12), vol. 1482 of AIP Conference Proceedings, pp. 464-470, 2012.

[11] A. U. M. Alkouri and A. R. Salleh, "Complex atanassov's intuitionistic fuzzy relation," Abstract and Applied Analysis, vol. 2013, Article ID 287382, 18 pages, 2013.

[12] D. Molodtsov, "Soft set theory-first results," Computers \& Mathematics with Applications, vol. 37, no. 4-5, pp. 19-31, 1999.

[13] P. K. Maji, A. R. Roy, and R. Biswas, "An application of soft sets in a decision making problem," Computers \& Mathematics with Applications, vol. 44, no. 8-9, pp. 1077-1083, 2002.

[14] P. K. Maji, R. Biswas, and A. R. Roy, "Soft set theory," Computers \& Mathematics with Applications, vol. 45, no. 4-5, pp. 555-562, 2003.

[15] P. K. Maji, R. Biswas, and A. R. Roy, “Fuzzy soft sets," Journal of Fuzzy Mathematics, vol. 9, no. 3, pp. 589-602, 2001.

[16] P. K. Maji, R. Biswas, and A. R. Roy, "Intuitionistic fuzzy soft sets," The Journal of Fuzzy Mathematics, vol. 9, no. 3, pp. 677692, 2001.

[17] P. K. Maji, R. Biswas, and A. R. Roy, "On intuitionistic fuzzy soft sets," Journal of Fuzzy Mathematics, vol. 12, no. 3, pp. 669-683, 2004.

[18] D. Pei and D. Miao, "From soft sets to information systems," in Proceedings of the IEEE International Conference on Granular Computing, vol. 2, pp. 617-621, July 2005.

[19] D. Chen, E. C. Tsang, D. S. Yeung, and X. Wang, "The parameterization reduction of soft sets and its applications," Computers \& Mathematics with Applications, vol. 49, no. 5-6, pp. 757-763, 2005.

[20] P. Majumdar and S. K. Samanta, "Generalised fuzzy soft sets," Computers \& Mathematics with Applications, vol. 59, no. 4, pp. 1425-1432, 2010.

[21] Y. Jiang, Y. Tang, H. Liu, and Z. Chen, "Entropy on intuitionistic fuzzy soft sets and on interval-valued fuzzy soft sets," Information Sciences, vol. 240, pp. 95-114, 2013.

[22] G. Zhang, T. S. Dillon, K. Y. Cai, J. Ma, and J. Lu, "Operation properties and $\delta$-equalities of complex fuzzy sets," International Journal of Approximate Reasoning, vol. 50, no. 8, pp. 1227-1249, 2009.

[23] P. Burillo and H. Bustince, "Entropy on intuitionistic fuzzy sets and on interval-valued fuzzy sets," Fuzzy Sets and Systems, vol. 78, no. 3, pp. 305-316, 1996. 


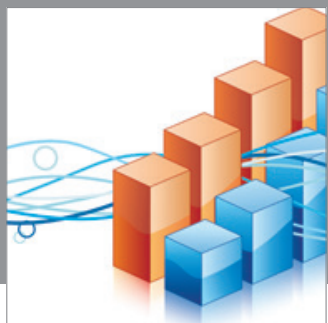

Advances in

Operations Research

mansans

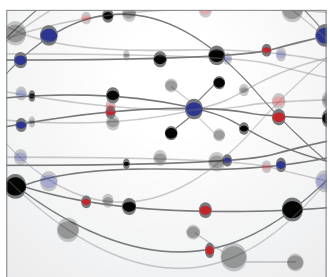

The Scientific World Journal
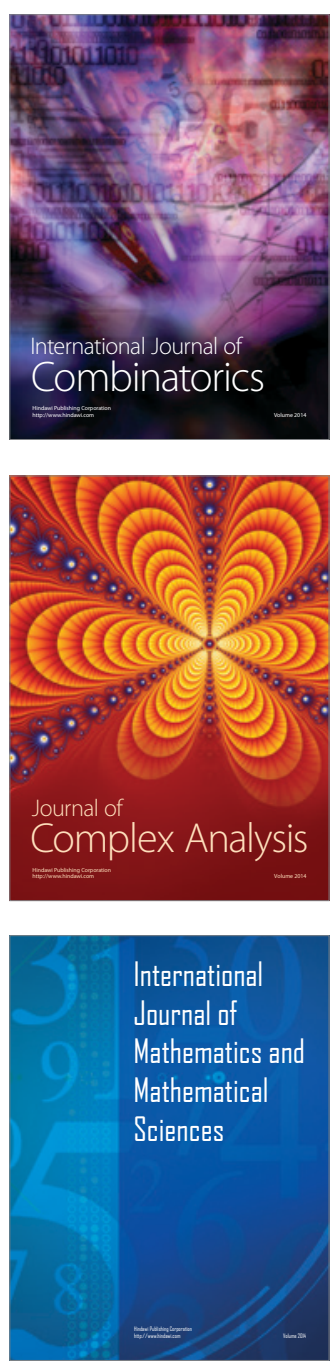
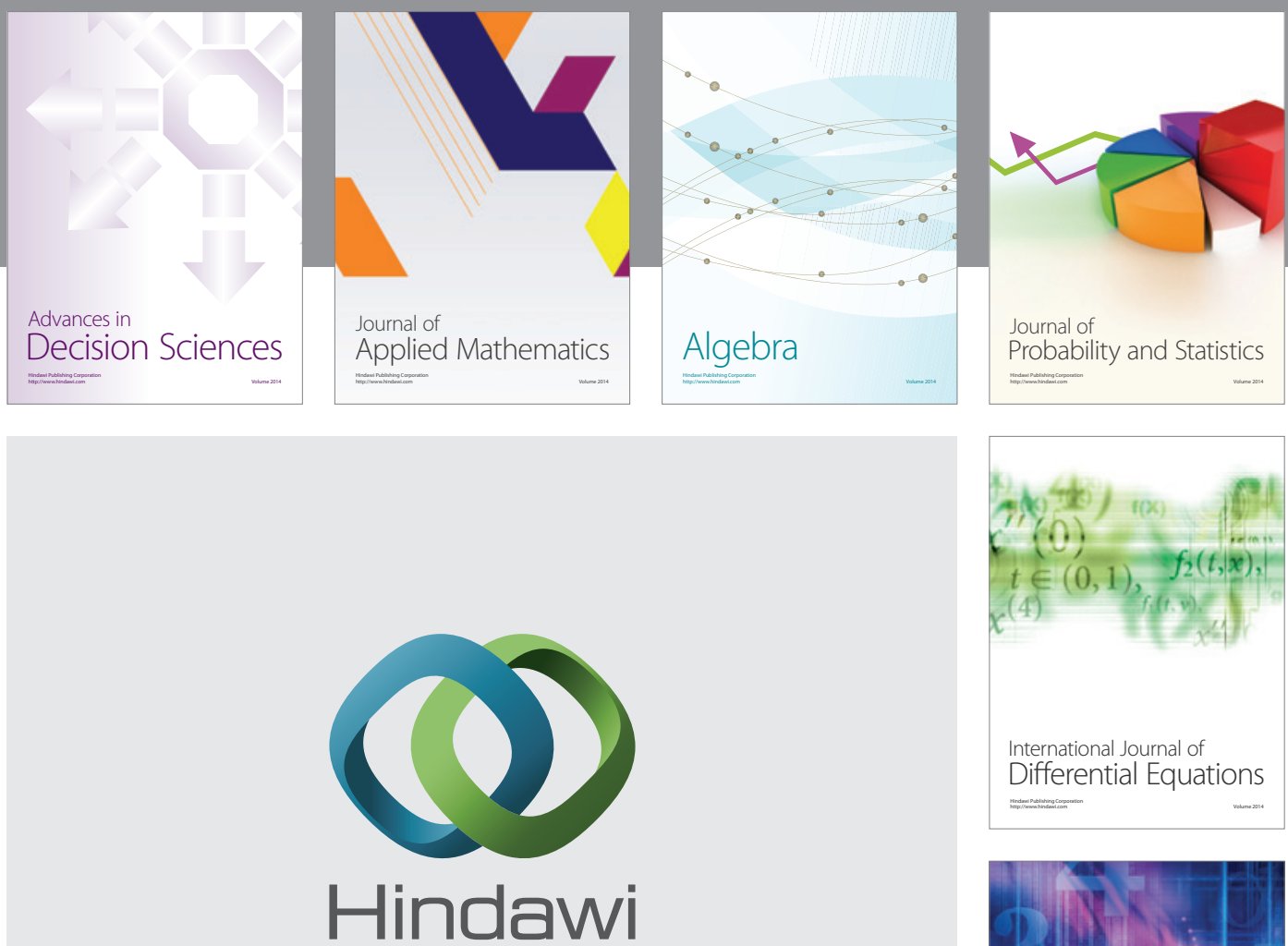

Submit your manuscripts at http://www.hindawi.com
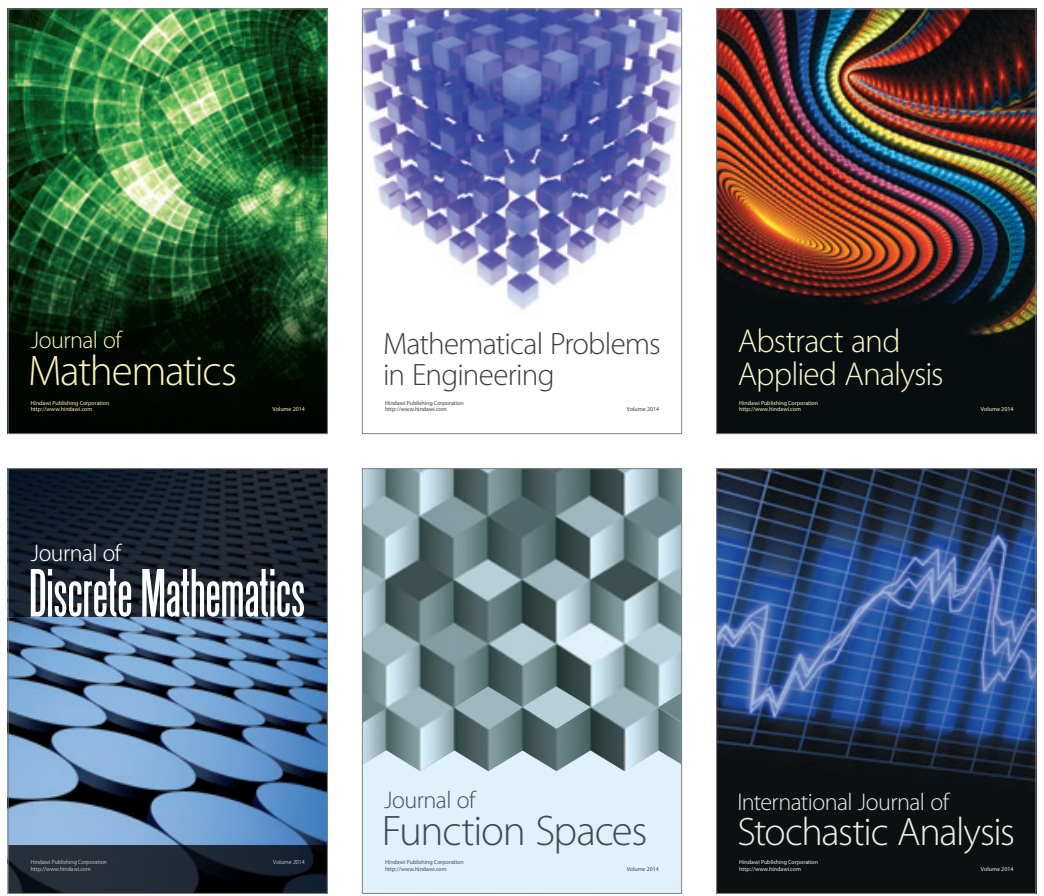

Journal of

Function Spaces

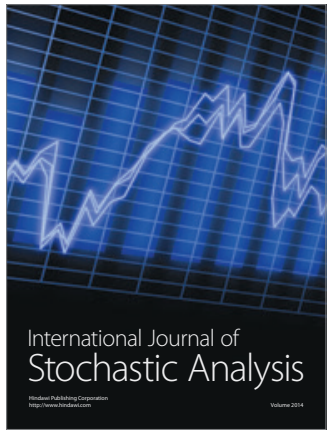

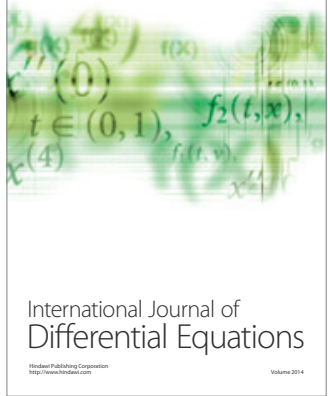
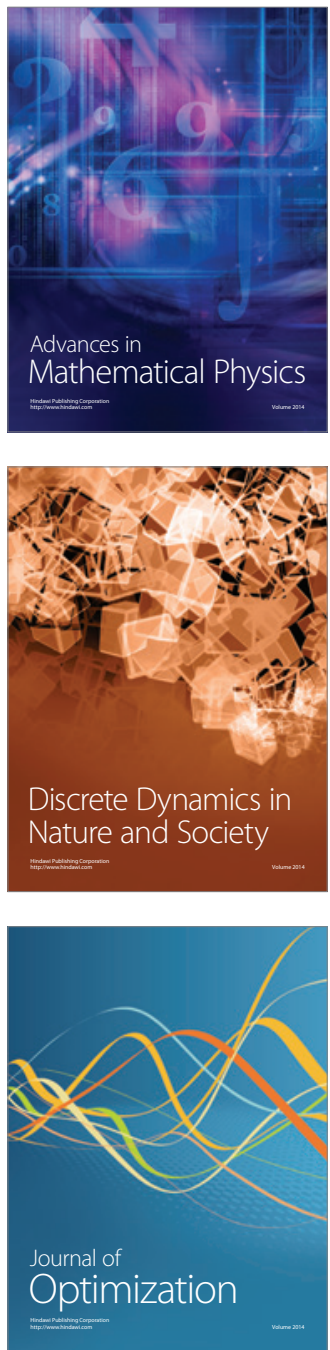